\title{
11 Title
}

2 DynaMiTES - a dynamic cell culture platform for in vitro drug testing PART 2 -

3 Ocular DynaMiTES for drug absorption studies of the anterior eye

\section{Authors}

$5 \quad$ Nicole Beißner ${ }^{1,3^{*}}$, Kai Mattern ${ }^{2,3^{*}}$, Andreas Dietzel ${ }^{2,3}$, Stephan Reichl ${ }^{1,3}$

61 Institut für Pharmazeutische Technologie, Technische Universität

7 Braunschweig, Mendelssohnstraße 1, 38106 Braunschweig, Germany

82 Institut für Mikrotechnik, Technische Universität Braunschweig,

9 Alte Salzdahlumer Straße 203, 38124 Braunschweig, Germany

103 Center of Pharmaceutical Engineering - PVZ, Technische Universität

11 Braunschweig, Franz-Liszt-Straße 35 A, 38106 Braunschweig

12 * These authors contributed equally to this work

\section{Address for Correspondence}

14 PD Dr. Stephan Reichl, Institut für Pharmazeutische Technologie, Technische

15 Universität Braunschweig, Mendelssohnstraße 1, 38106 Braunschweig, Germany

16 E-Mail: s.reichl@tu-braunschweig.de

17 Fon: 0531-391 5651

18 Fax: 0531-391 8108 


\section{Abstract}

In the present study, a formerly designed Dynamic Micro Tissue Engineering System (DynaMiTES) was applied with our prevalidated human hemicornea (HC) construct to obtain a test platform for improved absorption studies of the anterior eye (Ocular DynaMiTES). First, the cultivation procedure of the classic HC was slightly adapted to the novel DynaMiTES design. The obtained inverted HC was then compared to classic $\mathrm{HC}$ regarding cell morphology using light and scanning electron microscopy, cell viability using MTT dye reaction and epithelial barrier properties observing transepithelial electrical resistance and apparent permeation coefficient of sodium fluorescein. These tested cell criteria were similar. In addition, the effects of four different flow rates on the same cell characteristics were investigated using the

DynaMiTES. Because no harmful potential of flow was found, dynamic absorption studies of sodium fluorescein with and without $0.005 \%, 0.01 \%$ and $0.02 \%$ benzalkonium chloride were performed compared to the common static test procedure. In this proof-of-concept study, the dynamic test conditions showed different results than the static test conditions with a better prediction of in vivo data. Thus, we propose that our DynaMiTES platform provides great opportunities for the improvement of common in vitro drug testing procedures.

\section{Keywords}

DynaMiTES, human hemicornea construct, microfluidic system, organ on chip, TEER measurement, dynamic flow system, in vitro drug absorption, benzalkonium chloride 


\section{Introduction}

41

42

The development of novel drugs or innovative formulations is both cost intensive and time consuming. Thus, much effort is spent in the early phases of this process to obtain reliable findings. Unfortunately, most common preclinical test systems are based on animal experiments, ex vivo or in vitro models utilizing cell cultures, which all have well-known disadvantages. For example, the application of animals is ethically questionable and rarely representative for the effect in humans $[5,21,40]$. Furthermore, ex vivo as well as in vitro experiments are usually performed under static conditions, which cannot adequately mimic the physiological conditions in humans. Consequently, misleading outcomes often result in late stage drug failure. For this reason, scientists have been working for decades on improved in vitro methods for the early and reliable selection of the most important and promising drug candidates. One tremendous innovation in this field was the development of organ on chip (OOC) systems in the early 2010 s $[3,22,55]$. In these microstructured systems, well-characterized cell cultures can be cultured within small dimensions under a highly controlled microenvironment. These features should ease the in vitro emulation of human physiology and may ultimately lead to more reliable results $[5,10,32,49]$.

Despite great hope, OOC systems also exhibit essential disadvantages. On the one hand, they are poorly compatible with well-known cell culture systems. Thus, cell culture laboratories, which have long-term experience with in vitro models, cannot easily adapt them to OOC systems. Consequently, new efforts must be made to this purpose. Other dynamic systems, which enable the integration of standard cell culture models, such as the Quasi Vivo ${ }^{\circledR}$ system (Kirkstall, Rotherham, UK), do not provide additional analysis, such as continuous transepithelial electrical 
resistance (TEER) measurement [26], as OOCs try to include [6]. On the other hand, the most commonly used material for OOC fabrication, polydimethylsiloxane (PDMS), is known for its intensive substance absorption $[39,45]$. In research, it is used due to its easy structurability even with challenging geometries, its transparency, its biocompatibility and its gas permeability. However, substance absorption can hinder cell nutrition and, more importantly, the detection of drugs. For these reasons, PDMS is not an ideal material for routinely used absorption test systems of strong physiological and poorly permeated barriers, such as the human cornea.

Against this background, our interdisciplinary team of engineers and pharmacists has developed a microstructured system for dynamic in vitro drug absorption tests that circumvents these disadvantages and allows the integration of standard cell culture inserts and includes continuous TEER measurement. The resulting Dynamic Micro Tissue Engineering System (DynaMiTES; see Figure 1) was built for dynamic cell cultivation and, more importantly, the dynamic drug absorption testing on physiological barriers.

One pharmaceutically challenging physiological barrier is the human cornea. Ocular drug absorption is hindered to a great extent due to very tight cell-cell connections and short precorneal residence time because of tear flow $[4,23]$. Unfortunately, common ocular test systems apply static test conditions and thus fail to reliably emulate the dynamic human physiology. Consequently, the harmful effects of excipients, such as preservatives, might have a stronger effect on the permeated drug amount than in the clinical application. As no dynamic setup for cell culture models has been reported to date, an Ocular DynaMiTES for the implementation of in vivo-like conditions in pharmaceutical formulation testing would be the first step toward significantly improving the common test practice. Unlike static test systems, 
the enhanced dynamic system might not overestimate increased drug absorption resulting from non-physiological harmful conditions or toxic effects. For this reason, a dynamic test procedure will ensure an improved emulation of the in vivo situation and may offer a significant contribution to more reliable preclinical in vitro test systems [37].

For the generation of an Ocular DynaMiTES, our well-characterized and prevalidated human hemicornea $(\mathrm{HC})$ construct $[19,20]$ was applied within the DynaMiTES. In our first study, the technical design was tested and simulated intensively. Furthermore, the cell compatibility of all applied materials was investigated (see PART 1). Based on these findings, our second study (PART 2) focused on the cellular component the $\mathrm{HC}$ construct. First, the cultivation of the $\mathrm{HC}$ had to be slightly adapted due to the DynaMiTES design. The resulting inverted $\mathrm{HC}$ was then compared to the classic $\mathrm{HC}$ with regard to cell morphology, cell viability, TEER value and apparent permeation coefficient $\left(\mathrm{P}_{\mathrm{app}}\right)$ of sodium fluorescein as a paracellular marker substance to ensure similar cell characteristics. Second, absorption studies with sodium fluorescein were performed within the DynaMiTES with and without flow conditions. In this manner, potential harming or biasing effects of the materials and the engineering design could be examined. For this reason, four different flow rates were tested concerning cell viability, cell morphology, TEER value and $P_{a p p}$. After successfully performing this stepwise alteration of the common static in vitro practice, the first donor dilution experiments with and without a common ocular permeation enhancing preservative, benzalkonium chloride $(0.005 \%, 0.01 \%$ and $0.02 \%)[9,24,41]$, were performed to demonstrate the benefits of our dynamic test system. Taken together, these studies should show the importance and readiness of the Ocular DynaMiTES for application in improved drug absorption studies of the anterior eye. 


\section{Materials and Methods}

116

117 Cell culture flasks and Transwell $^{\circledR}$ inserts (art. no. 3402) were purchased from

119 respectively. The Keratinocyte Growth Medium (KGM), which emerged from the 120 Keratinocyte Basal Medium (KBM) by the addition of SingleQuots ${ }^{\circledR}$, was provided

121 from Lonza (Basel, Switzerland). $\mathrm{KCl}$ and $\mathrm{MgSO}_{4} \times 7 \mathrm{H}_{2} \mathrm{O}$ were acquired from Acros

122 Organics (Geel, Belgium). Phosphate Buffered Saline (PBS) tablets and EDTA 123 disodium salt solution were obtained from MP Biomedicals (Santa Ana, California, 124 US). Trypsin-EDTA and trypsin inhibitor were acquired from Thermo Fisher 125 Scientific (Waltham, Massachusetts, US). The acetic acid, $\mathrm{NaCl}, \mathrm{NaHCO}_{3}, \mathrm{HEPES}$, 126 ascorbic acid, D-glucose monohydrate, $\mathrm{CaCl}_{2} \times 2 \mathrm{H}_{2} \mathrm{O}$, sodium dodecyl 127 sulfate (SDS), formaldehyde $4 \%$ solution, glutaraldehyd $25 \%$ solution, 128 osmiumtetroxid $2 \%$ solution and hematoxylin solution were purchased from Carl 129 Roth (Karlsruhe, Germany). Technovit 7100 was obtained from Heraeus 130 Kulzer (Wehrheim, Germany). The $\mathrm{NaH}_{2} \mathrm{PO}_{4} \times \mathrm{H}_{2} \mathrm{O}$ and the L-glutamine were 131 provided by Merck (Darmstadt, Germany). Sodium fluorescein, hydrochloric acid, 132 10-fold MEM and 3-(4,5-Dimethyl-2-thiazolyl)-2,5-diphenyl-2H-tetrazolium 133 bromide (MTT) were purchased from Sigma-Aldrich (Munich, Germany). 134 Benzalkonium chloride (BAC) $50 \%$ solution was purchased from Caelo (Hilden, 135 Germany). The PCR mycoplasma test kit for routinely mycoplasma screening was 136 bought from Promocell (Heidelberg, Germany). The collagen for the three137 dimensional gel was extracted from rat tail following a standard protocol. The 138 employed Krebs-Ringer buffer (KRB) contained $6.8 \mathrm{~g} \mathrm{NaCl}, 0.4 \mathrm{~g} \mathrm{KCl}, 0.14 \mathrm{~g}$ 
$139 \mathrm{NaH}_{2} \mathrm{PO}_{4} \times \mathrm{H}_{2} \mathrm{O}, 2.1 \mathrm{~g} \mathrm{NaHCO}_{3}, 3.575 \mathrm{~g}$ HEPES, $1.1 \mathrm{~g}$ D-glucose monohydrate, $140 \quad 0.2 \mathrm{~g} \mathrm{MgSO}_{4} \times 7 \mathrm{H}_{2} \mathrm{O}$ and $0.26 \mathrm{~g} \mathrm{CaCl}_{2} \times 2 \mathrm{H}_{2} \mathrm{O}$ in $1000 \mathrm{~mL}$ of double-distilled water.

\subsection{Cultivation of Human Corneal Epithelial (HCE-T) Cells}

142 The HCE-T cell line originated from a 49-year-old female and was immortalized by 143 transfection with a recombinant SV40-adenovirus vector. This establishment and the 144 subsequent characterization were performed by Araki-Sasaki et al. [2]. The HCE-T 145 cells for the following study were purchased from the RIKEN cell bank (Tsukuba, 146 Japan) and cultured with $\mathrm{KGM}$ at $37{ }^{\circ} \mathrm{C}$ and $5 \% \mathrm{CO}_{2}$ in a humidified atmosphere.

\section{$147 \quad 7.3$ Cultivation of Human Corneal Keratocytes (HCK) Cells}

148 The HCK cell line was also immortalized by transfection with an SV40-adenovirus 149 vector but derived from human corneal keratocytes [20,57]. For the cultivation of our $150 \mathrm{HC}, \mathrm{HCK}$ cells were provided by Dr. M. Zorn-Kruppa (Hamburg, Germany) and 151 cultured with KGM under the same conditions as HCE-T cells.

152 Both cell types were routinely tested for mycoplasma infection.

\subsection{Cultivation of Human Hemicornea (HC) Construct}

154 For the combination of our well-known HC construct and novel DynaMiTES as an 155 Ocular DynaMiTES, the cultivation of $\mathrm{HC}$ was slightly adapted. Due to the insert orientation within the DynaMiTES (see Figure 2) and to ensure fluid flow over the corneal epithelium, HCE-T cells were grown on the bottom side of the insert 158 membrane. For this reason, the HC for the DynaMiTES was cultured inversely in 159 contrast to the classic culture conditions of Hahne et al. [19,20] (see Figure 3).

160 For the classic HC, HCK cells within a collagen stroma matrix and HCE-T cell lines 161 placed on top of the HCK cells were cultured with KGM under serum-free conditions 162 on permeable polycarbonate Transwell ${ }^{\circledR}$ filters at $37{ }^{\circ} \mathrm{C}$ and $5 \% \mathrm{CO}_{2}$ in a humidified 
163 atmosphere as described by Hahne et al. $[19,20]$. For the inverted HC, the collagen gel production was identical to the classic $\mathrm{HC}$ but different from the classic cultivation the Transwell ${ }^{\circledR}$ inserts were placed upside down into a 6-well plate after the gelling process. A HCE-T cell suspension of 100,000 cells/200 $\mu \mathrm{L}$ was then applied to the bottom surface of every insert. After cell adhesion for $1 \mathrm{~h}, 3 \mathrm{~h}$ or overnight (18 h), the inserts were reversed and cultured until day 7 similar to the classic $\mathrm{HC}$ protocol. On day 7 , all HCs were lifted to an air-liquid interface to induce an epithelial multilayer. The inverted $\mathrm{HCs}$ were once more inverted to the 6-well plate and fed daily with KGM from underneath the insert. For optimal cell nutrition, the inserts were always carefully tilted to avoid air accumulation under the collagen gel.

\subsection{Technical Design of the DynaMiTES}

As the technical component of our ocular system, the DynaMiTES consist of three separate levels: bottom level, insert level and top level (see Figure 1; for technical development and fabrication see PART 1). All these levels are built from polycarbonate (PC). Because the DynaMiTES was designed for repeated use, unlike common OOCs, the PC fabrication ensures enhanced durability and low substance absorption [51]. Furthermore, the modular design with its three levels enables individualization for a wide range of cell culture laboratories and their in vitro models. For example, the insert level can be adapted to different insert geometries, whereas the top and bottom levels, which are more elaborate to produce, can remain unchanged. The customizable insert level also consists of a sampling point to access the acceptor, which is situated between the insert and bottom levels. Acceptor and donor volumes were designed similar to common in vitro tests, at $1600 \mu \mathrm{L}$ and $370 \mu \mathrm{L}$, respectively. Furthermore, the microstructured donor channel with its posts ensures optimal mixing conditions (for channel construction and flow simulation see 
PART 1). However, this design is the opposite of the common test procedure, where

189 the donor is applied in the insert and the acceptor is provided beneath the insert (see

190 Figure 2 and Figure 6). This change was important to ensure in vivo-like fluid flow

191 across the cellular surface. Additionally, it eases dynamic donor variation, such as

192 donor dilution, mimicking physiological concentration gradients. Moreover, it allows

193 further downsizing of the channel dimensions for subsequent improvements to even

194 closer emulate human physiology. In addition to donor variation, TEER electrodes

195 built from stainless steel were included in the top and bottom levels. These additions

196 allow continuous TEER measurement and provide real-time information of the

197 substance-barrier interaction.

198 After successfully testing the cell compatibility (see PART 1) and cell characteristics 199 of the inverted HC (see Section 8.1), flow conditions were simulated using the 200 DynaMiTES. A basal flow rate $(31 \mu \mathrm{L} / \mathrm{min})$ and an initial flow rate $(73 \mu \mathrm{L} / \mathrm{min})$, 201 whereby the latter emulates the increased tear flow after instillation, were chosen 202 following Sörensen and Taagehöj [43] and calculated for the donor volume of $203370 \mu \mathrm{L}$ (see PART 1). In addition, a slightly elevated flow rate $(93 \mu \mathrm{L} / \mathrm{min})$ and 204 doubled initial flow rate $(148 \mu \mathrm{L} / \mathrm{min})$ were applied to investigate method limits. The 205 effect of these flow rates on cell morphology, cell viability, TEER and $P_{\text {app }}$ was 206 investigated using the following methods. As a negative control, inverted $\mathrm{HC}$ in the 207 standard well plate $(0 \mu \mathrm{L} / \mathrm{min}$ well plate) and in the DynaMiTES without 208 flow $(0 \mu \mathrm{L} / \mathrm{min}$ DynaMiTES) were investigated in parallel. 


\subsection{Cell Morphology}

210

211

212

213

\subsubsection{Light Microscopy (LM)}

For light microscopic observation of tissue cross sections using an Olympus IX50 photomicroscope (Olympus, Hamburg, Germany), the HCs were fixed with a phosphate buffered solution of $4 \%$ formaldehyde for $24 \mathrm{~h}$ and subsequently dehydrated with an ethanol series. Next, the HCs were cut into two halves, and each half was embedded in a plastic resin on a base of hydroxyethylmethylacrylate (Technovit 7100) according to the manufacturer's protocol. This resin block was cut in 3.5- $\mu \mathrm{m}$-thick slices, which were stained using hematoxylin and eosin.

\subsubsection{Scanning Electron Microscopy (SEM)}

For electron microscopic observation of the epithelial surface area using LEO 1550 (Zeiss, Oberkochen, Germany), the HCs were fixed with a $4 \%$ solution of glutaraldehyde for 90 min and post-fixed with a $2 \%$ solution of osmium tetroxide for $2 \mathrm{~h}$. Subsequently, the tissue was dehydrated employing an ethanol series and air-dried overnight. After this drying process, the Transwell ${ }^{\circledR}$ membrane was immobilized on an SEM sample holder, and the epithelial surface was coated with gold using Bal-Tec SCD 050 (Balzer, Wiesbaden, Germany).

For the morphological evaluation of flow effects, inverted HCs in the standard well plate $(0 \mu \mathrm{L} / \mathrm{min}$ well plate $)$ and in the DynaMiTES without flow $(0 \mu \mathrm{L} / \mathrm{min}$ DynaMiTES $)$ served as a negative control. In contrast, inverted HCs whose epithelium was scratched with a glass pipette were treated equally as a positive reference.

\subsection{Cell Viability}

Cell viability was investigated by using an MTT dye reaction. For this purpose, the apical and basolateral side of the HC were exposed to a $1+4$ mixture of MTT stock 
233 solution $(5 \mathrm{mg} / \mathrm{mL})$ and $\mathrm{KGM}$. After three hours of reaction at $37^{\circ} \mathrm{C}, 5 \% \mathrm{CO}_{2}$ and 234 humidified atmosphere, the MTT solution was removed. For cell lysis, a lysis solution 235 containing $2.73 \mathrm{~g}$ SDS, $3.64 \mathrm{~g}$ hydrochloric acid, $88.18 \mathrm{~g}$ water and $905.45 \mathrm{~g}$ 236 isopropanol was applied to both sides of the HC and incubated overnight. Next, an 237 aliquot was diluted 1+3 with fresh lysis solution, and absorption was measured at $238570 \mathrm{~nm}$ using an UV-VIS plate reader Powerwave XS from BioTek (Winooski, 239 Vermont, US). On the basis of these results, cell viability was calculated in 240 relationship to an untreated reference in a well plate.

241 To investigate the effect of flow, the inverted HCs were set into the DynaMiTES insert 242 level. Next, different flow rates of KRB were applied for the intended experimental 243 duration of three hours. Cell viability was then assessed as previously described.

\subsection{Transepithelial Electrical Resistance}

TEER measurement during cultivation, as well as prior to and during the absorption

246 studies, was performed using EVOM $^{\circledR}$ combined with Endohm ${ }^{\circledR}$ Chamber from World 247 Precision Instruments (WPI, Sarasota, Florida, US). For TEER measurement within 248 the DynaMiTES, the incorporated electrodes were connected to the $\mathrm{EVOM}^{\circledR}$, and 249 TEER was measured on every sampling point of the absorption study. For the TEER 250 profiles, the starting TEER value of every $\mathrm{HC}$ was set as $100 \%$, and the subsequent 251 values were calculated in relationship to the starting value.

252 As a negative control, the TEER profiles of inverted HCs in a normal well plate were 253 assessed simultaneously for each experiment. Biasing effects on the TEER 254 measurement resulting from the DynaMiTES construction or the flow itself were 255 investigated with a positive control. For this reason, inverted HCs were devitalized 256 with a solution of $0.03 \%$ SDS over $3 \mathrm{~h}$ prior to the experiment. Subsequently, the 
257 TEER profiles were analyzed over $180 \mathrm{~min}$ with a flow rate of $31 \mu \mathrm{L} / \mathrm{min}$. Because no 258 base line shift was observed, biasing effects of the DynaMiTES cannot be 259 expected (data not shown).

\subsection{Static Absorption Studies}

261 In addition to TEER, the absorption of sodium fluorescein as a hydrophilic and 262 paracellular marker substance [31] was used for the evaluation of epithelial barrier 263 properties. All absorption studies were performed in KRB according to the 264 prevalidation study of the classic HC [20]. The donor solution $(250 \mu \mathrm{g} / \mathrm{mL}$ sodium 265 fluorescein in KRB) was applied to the epithelial surface at day 10 of cultivation (see 266 Figure 6A), and the amount of permeated sodium fluorescein in the acceptor 267 compartment was detected over the course of $180 \mathrm{~min}$. All acceptor samples were 268 analyzed using a fluorescence microplate reader Genios from Tecan (Männedorf, 269 Switzerland) at an excitation wavelength of $485 \mathrm{~nm}$ and an emission wavelength of $270535 \mathrm{~nm}$. From the obtained concentrations, the amount of permeated sodium

271 fluorescein was calculated and plotted over time. The linear ascent of these 272 permeation profiles was then used to calculate the apparent permeation 273 coefficient $\left(P_{\text {app }}\right)$ as described by Hahne et al. [20].

274 For absorption studies within the DynaMiTES, the inlet of every system was 275 connected with a syringe pump (KDS 270 Legacy Syringe Pump, KD Scientific, 276 Holliston, Massachusetts, US). The donor compartment was then either filled once 277 with the donor solution $(250 \mu \mathrm{g} / \mathrm{mL}$ sodium fluorescein) for the simulation of classic 278 static test conditions or the donor was pumped along the epithelial surface with four 279 different flow rates for flow effect investigation (see Figure 2 and Section 7.5). 


\subsection{Static vs. Dynamic Absorption Studies}

281 The dynamic absorption studies were performed with a 10-fold higher concentration 282 of sodium fluorescein $(2.5 \mathrm{mg} / \mathrm{mL})$ to ensure acceptor concentration above the 283 detection limit. After a single donor filling (bolus application), an initial flow rate of $284 \mathrm{KRB}(73 \mu \mathrm{L} / \mathrm{min})$ was applied for $5 \mathrm{~min}$ to simulate elevated tear drainage after 285 instillation $[33,43]$. Subsequently, a basal flow of $\mathrm{KRB}(31 \mu \mathrm{L} / \mathrm{min})$ was applied for $175 \mathrm{~min}$. Acceptor samples were obtained and analyzed similar to the static absorption studies. In parallel, static experiments without KRB dilution were performed in the DynaMiTES with the same donor solution.

As a proof-of-concept, the comparison of static and dynamic test conditions was further performed after exposure to $0.005 \%, 0.01 \%$ and $0.02 \%$ BAC in KRB. To 291 avoid BAC and sodium fluorescein interaction, both substances were applied 292 consecutively. In the first phase, the HC was exposed to a specific BAC solution. For 293 dynamic exposure, BAC was diluted by $\mathrm{KRB}$ flow $(5 \mathrm{~min} 73 \mu \mathrm{L} / \mathrm{min}$ and $25 \mathrm{~min}$ $29431 \mu \mathrm{L} / \mathrm{min}$ ) after a bolus application. A second flow regime with $10 \mathrm{~min}$ of $73 \mu \mathrm{L} / \mathrm{min}$ 295 and $20 \mathrm{~min}$ of $31 \mu \mathrm{L} / \mathrm{min}$ was tested with the highest BAC concentration to 296 investigate the flow rate effect $(0.02 \%$ EF = elevated flow). In static experiments, the 297 donor remained unchanged for $30 \mathrm{~min}$. In the second phase, the sodium fluorescein 298 permeation was performed similar to the dynamic absorption studies over the course 299 of 180 min. Dynamic BAC exposure was always followed by dynamic sodium 300 fluorescein permeation to simulate the novel dynamic test conditions. In contrast, 301 static BAC exposition was followed by static sodium fluorescein permeation to 302 recreate common static test procedures. For the latter, the $\mathrm{P}_{\mathrm{app}}$ was calculated from 303 the linear ascent as previously described (see Section 7.9). In the dynamic 304 experiments, this was not feasible due to the lack of infinite dose. Thus, the acceptor 
concentrations of sodium fluorescein were utilized for a comparison of the dynamic conditions.

\subsection{Statistical Analysis}

Statistical analysis was performed using IBM SPSS Statistics 24 (IBM, Armonk, New York, US). Differences between two groups were compared using independent twosample t-test. More than two groups were investigated using one-way ANOVA (analysis of variance). For detailed distinction, of differences found in ANOVA the Bonferroni post hoc test was used for homogeneous variance and the Games-Howell post hoc test for inhomogeneous variances. p-values of less than 0.05 were considered significant $\left(p<0.05^{\star}, p<0.01^{\star *}\right)$.

\section{Results}

\subsection{Adaption of HC Cultivation to the DynaMiTES Design}

As previously discussed, the $\mathrm{HC}$ cultivation was adapted for the application of the $\mathrm{HC}$ construct within the DynaMiTES. The resulting inverted $\mathrm{HC}$ were then compared to the classic $\mathrm{HC}$ with regard to cell morphology, cell viability, TEER and $\mathrm{P}_{\mathrm{app}}$ to ensure similar cell characteristics.

\subsubsection{Cell Morphology}

A variation in attachment time $(1 \mathrm{~h}, 3 \mathrm{~h}$ und $18 \mathrm{~h})$ for the epithelial cells of the inverted $\mathrm{HC}$ displayed an increasing number of epithelial layers with increasing attachment time (see Table 1). However, morphology similar to the classic HC was best obtained with overnight attachment. Classic as well as inverted $\mathrm{HC}$ showed both tight epithelial cell growth with two to more cell layers under these conditions (see Figure 3). Thus, overnight attachment was used for all following experiments. 


\subsubsection{Cell Viability}

329 Cell viability of classic and inverted HC were compared using MTT dye reaction. As a 330 positive reference, a group of stroma equivalents without HCE-T cells and only 331 containing HCK cells was cultured simultaneously. On day 10 of cultivation, classic 332 as well as inverted $\mathrm{HC}$ showed cell viabilities of or close to $100 \%$, which were not 333 significantly different from each other. In contrast, the cell viability of the positive 334 reference was significantly diminished, caused by HCE-T cell exclusion (see 335 Figure 4).

\subsubsection{Transepithelial Electrical Resistance}

In addition to cell morphology and cell viability, TEER values were measured on

338 cultivation day 6 , the day before ALI (see Figure 3), and until day 13. In this manner, 339 changes in the epithelial barrier resulting from the ALI cultivation could be 340 investigated over an extended period of eight days (see Figure 5). Within this time 341 span, the classic HC showed higher TEER values at the beginning, which decreased constantly during ALI cultivation. In contrast, the inverted HC showed decreasing 343 TEER values, which increased after the beginning of ALI cultivation. Despite these 344 differing TEER developments in the early phases of cultivation, classic as well as 345 inverted HC showed similar TEER values on day 10, the day of application for 346 absorption studies following Hahne et al. [20]. An extended cultivation did not show any significantly different TEER values for the following three days. A TEER optimum was reached between day 10 and day 11 for the inverted $\mathrm{HC}$, which was similar to

349 the findings observed by Hahne and Reichl for classic HC in 2011 [19].

\subsubsection{Absorption Studies}

351 Following the absorption studies of Hahne et al. [19,20], the $P_{\text {app }}$ of sodium 352 fluorescein was analyzed on day 10 of cultivation for the inverted as well as for the 
353 classic $\mathrm{HC}$. As the similar TEER values might have indicated, the obtained $\mathrm{P}_{\text {app }}$ 354 values were also not significantly different between both groups. Moreover, the 355 values were similar to the values for the classic $\mathrm{HC}$ published by 356 Hahne et al. [20] (see Figure 6).

\subsection{Absorption Studies with and without Flow in the DynaMiTES}

358 After successfully adapting the HC's cultivation for the novel design, the potential 359 harmful effects of fluid flow should be investigated prior to the application of dynamic 360 experimental test conditions. For this reason, cell morphology, cell viability, TEER 361 and $\mathrm{P}_{\mathrm{app}}$ of the inverted $\mathrm{HC}$ were analyzed with and without utilizing different flow 362 rates.

\section{$363 \quad$ 8.2.1 Cell Morphology}

364 Cell morphology was observed using LM for the investigation of cell abrasion or 365 alterations in cell layer formation. Furthermore, SEM was used for an extensive 366 observation of the epithelial surface to detect randomly occurring changes in the 367 epithelium.

368 In LM, neither cell detachment nor hole formation as a consequence of the applied 369 fluid flows were observed (see Table 2; left column). All images showed a 370 multilayered epithelium with a more or less uneven surface, which may have resulted 371 from the loss of contact inhibition as previously demonstrated for other SV-40 372 immortalized cell lines $[30,44]$ or the fact that in contrast to the in vivo situation all cell 373 layers are proliferating. Furthermore, the lack of lid movement and desquamation 374 may have caused a less smooth and compact surface. However, all appearances in 375 the DynaMiTES regardless of flow were similar to the negative control in the well 
plate. Moreover, a clear scratch in the deliberately injured positive control was visible and demonstrated that changes in cell morphology would be detectable.

378 In the SEM images, a similar observation could be obtained (see Table 2, right

379 column). The samples with and without flow from the DynaMiTES showed no hole 380 formation, as no PC membrane was visible in any of the samples areas. However, an 381 intended injury, and thus the underlying PC membrane were clearly visible in the 382 positive control. By locating the injury close to the margins, we were able to 383 demonstrate that it is possible to detect mechanical injuries close to the insert edges 384 using SEM imaging. Regarding the cellular surface, uneven cell areas were observed 385 similar to the LM images. Overall, only a slight tendency toward a more defined surface structure could be observed with an increasing flow rate. The irregular surface in the image of the flow rate $(93 \mu \mathrm{L} / \mathrm{min})$, which was not observed in other 388 samples, may be caused from the sample preparation.

\subsubsection{Cell Viability}

In addition to cell morphology, cell viability was examined after three hours of flow exposition. Because this was the expected experimental time for subsequent drug absorption studies, cell viability should not be restricted by shear stress or other effects during this period. A negative control was maintained in $\mathrm{KRB}$ in a standard well plate. Furthermore, a group of inverted HCs were inserted into the DynaMiTES, and no flow was applied $(0 \mu \mathrm{L} / \mathrm{min})$. The results in Figure 7 underline that no clear

396 reduction in cell viability was found as all groups showed values above $90 \%$ regardless of the tested flow rate.

\subsubsection{Transepithelial Electrical Resistance}

399 The integrated TEER electrodes of the DynaMiTES were used to investigate TEER development over the experimental time of $180 \mathrm{~min}$. Thus, the relative TEER value 
401 was obtained with and without flow over the course of $180 \mathrm{~min}$ (see Figure 8). As a negative control, the TEER development of inverted HCs in a cell culture well plate was analyzed simultaneously for every batch.

404 When comparing the inverted HCs, a slight but constant decreasing trend could be 405 observed in the negative control. Resulting from this decrease, all groups from the 406 DynaMiTES (except $73 \mu \mathrm{L} / \mathrm{min}$ ) were significantly different to $0 \mu \mathrm{L} / \mathrm{min}$ well 407 plate $(p<0.05)$ after $180 \mathrm{~min}$ but not to $0 \mu \mathrm{L} / \mathrm{min}$ DynaMiTES. Furthermore, a flow 408 rate of $73 \mu \mathrm{L} / \mathrm{min}$ showed a short decrease in TEER value within the first $10 \mathrm{~min}$ and 409 a stable value afterwards. All other flow rates initially showed ascending and subsequently stable TEER profiles. From these flow rates, the inverted HCs, which were exposed to $93 \mu \mathrm{L} / \mathrm{min}$, showed the highest relative TEER value.

\section{$412 \quad$ 8.2.4 Absorption Studies}

413 As a second parameter for the evaluation of epithelial barrier properties, the $\mathrm{P}_{\mathrm{app}}$ of 414 sodium fluorescein was accessed in the DynaMiTES with and without the application 415 of different flow rates. As a negative control, an absorption study in a well plate was 416 performed simultaneously in every experiment. The obtained $\mathrm{P}_{\text {app }}$ values are shown 417 in Figure 9. The inverted HCs from the DynaMiTES showed all and, regardless of the 418 flow no significant increase in $\mathrm{P}_{\mathrm{app}}$ compared to the reference in the well plate. Only 419 an injury, resulting from a static exposition to $0.01 \%$ BAC, had significantly increased 420 the determined $P_{\text {app }}$.

\section{8.3 Dynamic Absorption Studies in the DynaMiTES}

422 After the investigation of all harmful or interfering effects, the DynaMiTES was utilized

423 for a small proof-of-concept study. Because, the system should emulate physiological 424 tear drainage and thus enable the reliable evaluation of ocular drug absorption at the 
425 anterior eye, the effect of divers benzalkonium chloride concentrations were

426 investigated under static and dynamic experimental conditions.

\section{8.3.1 Static vs. Dynamic Absorption Studies}

428 The obtained results (see Figure 10) for sodium fluorescein without BAC addition 429 showed a typical linear concentration-time curve for the static test conditions after 430 reaching a steady state. In contrast, dynamic test conditions showed a lower curve, 431 which flattened until the end of the experiment.

432 Following exposure to $0.005 \%$ BAC, the curves for both conditions were similar to 433 the curves without BAC. For the concentration of $0.01 \% \mathrm{BAC}$, an increase in 434 permeability $\left(\mathrm{P}_{\mathrm{app}}\right)$ could only be detected for the static procedure $435\left(2.83 \times 10^{-7} \pm 0.09 \times 10^{-7} \mathrm{~cm} / \mathrm{s}\right.$ without $\mathrm{BAC}$ vs. $12.2 \times 10^{-7} \pm 6.91 \times 10^{-7} \mathrm{~cm} / \mathrm{s}$ for $4360.01 \%$ BAC - 4.3-fold increase in $\left.\mathrm{P}_{\mathrm{app}}\right)$, whereas no increase was found under 437 dynamic conditions. When applying $0.02 \%$ BAC, the curves for static $438\left(12.6 \times 10^{-7} \pm 2.09 \times 10^{-7} \mathrm{~cm} / \mathrm{s}-4.5\right.$-fold increase in $\left.\mathrm{P}_{\mathrm{app}}\right)$ and dynamic conditions 439 (10.3-fold increase in concentration) were both increased compared to the curves 440 without BAC. Furthermore, the static curves for $0.01 \%$ and $0.02 \%$ BAC were 441 comparable and showed no further increase in sodium fluorescein concentration with 442 increasing BAC concentration. In contrast to the other curves, the two conditions both 443 showed dramatically shortened lag phases in the beginning and had flattened over 444 the entire experimental time.

445 However, the increase in dynamic concentration profile could not be observed for $446 \quad 0.02 \%$ BAC with the second flow regime involving an extended initial flow rate phase 447 (1.6-fold increase in concentration with $0.02 \%$ BAC EF = elevated flow). 


\section{Discussion}

449

450

451

452

453

454 468 time period examined in the study of Wakabayashi et al. [56]. Nevertheless, no 469 harming effects were detected in the MTT test, as the initial seeding volume of $470200 \mu \mathrm{L}$ was higher than the $100 \mu \mathrm{L}$ in the study of Wakabayashi et al. and our

\subsection{Adaption of HC Cultivation}

The four cell characteristics, morphology, viability, TEER and $P_{a p p}$, which were observed in this study together indicated that an alteration in the cultivation procedure did not significantly affect the tested criteria of our HC. The HCE-T cells on the bottom surface of the membrane developed an epithelium with 2-4 cell layers, suggesting similar epithelial barrier properties (TEER and $\mathrm{P}_{\mathrm{app}}$ ). Other inverted cultivations without prior coating have been previously described for Calu-3 cells and MDCK II cells in a lung model [7] and kidney model [56], respectively. However, to the best of our knowledge, cultivation on the bottom surface of the insert has not yet been reported for corneal cells, specifically HCE-T. A slightly different inverted cultivation has been published by Zorn-Kruppa et al. [57]. However, their cultivation of HCE-T cells within the insert does not allow direct flow exposition to the epithelium. For this reason our inverted cultivation is more suitable for the intended in vivo-like conditions within the DynaMiTES.

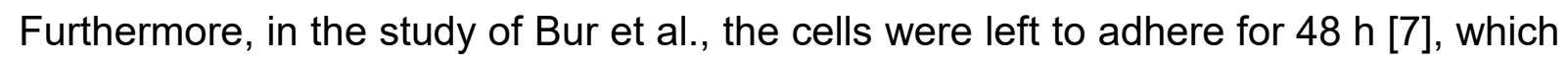
is longer than in our protocol, although a larger medium volume of $500 \mu \mathrm{L}$ (compared to $200 \mu \mathrm{L}$ ) was used. To apply this larger volume, tubes were placed over the insert. Advantageously, these tubes, which may harm the cell layer when removed, were avoided in our protocol. Moreover, our overnight adhesion was longer than the 5 -h 
471 collagen gel was generated using 10-fold MEM and thus ensured cell nutrition until 472 the next day.

473 Morphologic observations demonstrated that the two cell types could grow in physical 474 proximity. The cells might interact even through the PC membrane as the pore size 475 of $3 \mu \mathrm{m}$ is much wider than the molecule size of cytokines and other signal

476 molecules. For this reason, the benefit of co-cultivation remains unchanged and, 477 thus, other cell characteristics, such as substance transport [52-54] and substance 478 metabolism $[27,28]$, which we investigated extensively in our former studies, might 479 also be maintained. This finding was also supported by the cell viability results. 480 Classic and inverted HC showed both similar values. Only the value of the stromal 481 equivalents was higher than it might be estimated by the initial cell seeding 482 density [19]. However, this may result from a higher metabolic activity of the HCK 483 cells compared to the HCE-T cells.

484 With regard to the TEER observation, the differences in the early TEER development 485 between classic and inverted $\mathrm{HC}$ are of minor interest, as all models were not used 486 until day 10 of cultivation and upon completed formation of the epithelial multilayer. 487 Furthermore, the late TEER values, including the TEER optimum from day 10 to 488 day 11 as well as the $\mathrm{P}_{\text {app }}$ values were comparable between all groups and similar to 489 the values reported in previous studies $[19,20]$.

\subsection{Absorption Studies with and without Flow in the DynaMiTES}

491 Morphological investigation with and without flow did not result in cell detachment or 492 hole formation in the epithelium. Because we were able to detect injuries close to the 493 margins using SEM imaging, we could also conclude that insertion of our in vitro 494 model into the DynaMiTES did not cause any damage. This assumption was 
supported by other previous results. Extensive detachment of the cells would have 496 decreased the obtained cell viability as previously demonstrated for stromal 497 equivalents (see Figure 4 and Figure 7). Moreover, regardless of flow exposure, no 498 significant decrease in TEER or increase in $\mathrm{P}_{\text {app }}$ values occurred in the DynaMiTES. 499 Thus, no negative effects of the flow were expected. Nevertheless, the flow rates of 500 the current study were relatively low. Consequently, the shear 501 stresses $\left(1.72 \times 10^{-4} \mathrm{~Pa}\right.$ for $148 \mu \mathrm{L} / \mathrm{min}$; for shear stress calculation see PART 1$)$ 502 were much lower compared to the in vivo conditions (up to $15 \mathrm{~Pa}$ [15]). However, we 503 only aimed to simulate tear drainage and thus did not intend to apply shear stress 504 similar to physiological blinking forces. In the present work, the shear stresses and 505 short exposure time resulted in slight changes in cell morphology or TEER, which 506 might be extended with application of higher shear stresses and a prolongation of 507 flow exposure. Stimulating or life extending effects of physiological flow have for 508 example been reported for models of the human gut [25], liver and skin [50,55]. 509 Regarding monolayers of SV-40 immortalized corneal epithelial cell lines, 510 Pretor et al. found no morphological changes with a shear stress of $0.1 \mathrm{~Pa}$ [38]. 511 However, Utsunomiya et al. observed significantly decrease in wound healing and 512 corneal cell proliferation after the exposition to 1.2 Pa [48]. For this reason, an effect 513 of increased shear stress in drug absorption testing or specifically in dynamic 514 cultivation for the inverted HC (see Section 10) should be further investigated in 515 subsequent studies.

516 In addition to the investigation of flow effects, we showed the reliability and benefit of 517 the inbuilt TEER electrodes in real-time TEER assessment. Because standard 518 measuring systems, such as EVOM2 combined with EndOhm ${ }^{\circledR}$ Chamber, only allow 519 TEER analysis before and after the absorption study without any disruption, no real- 
520 time information with a time-dependent evaluation of substance barrier interactions is

521 feasible. Alternative measurement systems, such as the EVOM2 with chopstick 522 electrode (STX2 electrode, WPI, Sarasota, Florida, US), entail multiple error 523 possibilities as variable electrode positions and cross-contamination of the tested 524 substance. Fortunately, our DynaMiTES resolves all these challenges and enhances 525 the informational content that is obtained in in vitro absorption studies.

\section{9.3 Dynamic Permeation Studies in the DynaMiTES}

527 In our first dynamic experiments without BAC, the novel and in vivo-like test 528 procedure showed a reduced concentration profile compared to static conditions.

529 This finding resulted from shorter exposure time periods to sodium fluorescein in 530 consequence of the simulated tear drainage. Moreover, the dynamic curve flattened 531 over the permeation time due to of the diminishing concentration in the donor 532 compartment and the consequently decreasing concentration gradient. This 533 progression more resembled the physiological kinetics $[13,42]$ than static conditions. 534 In the latter, the high donor concentration generated a constant concentration 535 gradient during the entire experimental time. As a result, higher acceptor 536 concentrations and linear concentration-time curves were achieved under static 537 conditions. Although, these findings enable further calculation of the $P_{a p p}$ as a 538 parameter for substance absorption, this profile progression cannot be easily 539 transferred to in vivo conditions [46].

540 Apart from the differences in static and dynamic experimental conditions, we 541 investigated the effect of BAC. Because this substance is a common and frequently 542 used preservative, many studies examining the effects of BAC have been previously 543 performed. Among these studies, an increasing harmful potential with increasing 544 concentration has been reported $[11,18,36]$ and was also found in the present study. 
545 For example, the lowest BAC concentration $(0.005 \%)$ showed neither an effect 546 under static nor under dynamic conditions. The doubled concentration then caused a 547 clear effect only under static conditions (4.3-fold increase in $\mathrm{P}_{\text {app }}$ ), which could be 548 marginally increased with a higher BAC concentration. For $0.02 \%$ BAC, the $\mathrm{P}_{\text {app }}$ was 549 4.5-fold higher than the reference without BAC. In this case, the non-physiological 550 static exposure time of 30 min may have resulted in an immense and maximum 551 barrier-impairing effect for $0.01 \%$ and $0.02 \%$ BAC. Consequently, no further 552 increase in permeability was observed with increasing concentration. Moreover, this 553 large barrier disruption may have caused a dramatic reduction in lag time for sodium 554 fluorescein permeation under these conditions.

555 However, no increase in sodium fluorescein concentration was found for dynamic 556 conditions lower than $0.02 \%$ BAC. This high concentration then caused a 10.3-fold 557 increase in acceptor concentration compared to dynamic conditions without BAC. No 558 effect was found below $0.02 \%$ BAC, which was consistent with findings obtained in 559 the study performed by Burstein [8], who investigated sodium fluorescein 560 permeability in vivo (dynamic conditions). In their study, $0.01 \%$ BAC also showed no 561 significant increase. However, the permeability was increased 1.23 -fold by $0.02 \%$ of 562 BAC. The effect of $0.02 \%$ BAC was also investigated by Uematsu et al. [47] using an 563 in vivo TEER measuring system. A significant decrease in TEER was observed in 564 humans at $0.02 \%$ BAC but not at $0.01 \%$ BAC. Higher concentrations were not tested 565 due to their harmful potential [17]. Furthermore, both studies showed, that both 566 investigated rabbit groups were more susceptible to BAC and yielded significant 567 results at lower concentrations compared to humans $[8,47]$. These findings underline 568 once more that rabbits cannot reliably predict human physiology and that alternative 569 test systems are needed. 
570 Apart from concentration-dependent effects, exposure time-dependent effects have 571 also been reported for BAC [18,29,46]. For example, Nakagawa et al. [34] found no 572 significant increase in carboxyfluorescein permeability for $0.002 \%$ BAC after 10 min 573 exposure, but only after $6 \mathrm{~h}$ exposure. These investigations highlight that the results 574 are not only dependent on the BAC concentration but also on its exposure time. In 575 our experiments, $0.01 \%$ BAC significantly increased the concentration curve under 576 static conditions but not under dynamic conditions, as the exposure time was clearly 577 shorter and in vivo-like in the dynamic test system.

578 Because BAC is rapidly diluted in vivo [16], dynamic test conditions have always been favorable but rarely developed. Nakamura et al. [35] generated a dynamic ex vivo test system in which the rabbit cornea is mounted in an Ussing chamber system and the donor compartment is drained by a peristaltic pump. Similar to the previously

582 described studies, the decrease in TEER was higher with increasing BAC 583 concentration. More importantly, the turnover rate was varied to simulate normal or 584 dry eye precorneal physiology, and an increasing harmful potential with decreasing flow rate was observed. These findings were confirmed by our results, as we were able to nearly eliminate the barrier-impairing effect of $0.02 \%$ BAC with an elevated 587 flow rate (10.3-fold vs. 1.6-fold increase of sodium fluorescein concentration). The 588 importance of the chosen flow rate may also explain the relatively high increase in 589 acceptor concentration after dynamic exposure to $0.02 \%$ BAC for the normal flow rate. In our study, the experimental conditions resulted in a 10.3-fold increase.

591 However, Burstein only found a 1.23-fold increase in vivo [8]. Taking this study into 592 consideration, our elevated flow rate resulted in similar values compared to the in 593 vivo conditions, and a better in vivo correlation may be obtained with a slightly higher 594 flow rate. In the study of Nakamura et al. [35], a higher tear flow was selected in 
595

596

597

598 states.

\section{2}

617 transplantation and are rarely available for other applications, such as in studies 618 using the Ussing chamber system developed by Nakamura et al. For this reason, we 
are convinced that our DynaMiTES represents the optimal combination of a

620 prevalidated human in vitro model and a highly controlled dynamic modular device.

621 With its application in pharmaceutical substance testing, the disadvantages of animal

622 tissue-based test systems and static in vitro models may be circumvented. Finally, 623 automation, which is highly demanded in routine application, is realizable. The donor 624 application has been previously performed using programmable syringe pumps, and 625 the sampling points are provided with septa to enable automatic sampling similar to 626 HPLC autosamplers.

627 Taken together, these advances ease the application in pharmaceutical research and 628 development (R\&D). Possible and meaningful applications not only include use in 629 drug absorption but also in functional tests of additives, including permeation 630 enhancers and colloidal systems, such as micelles, liposomes and nanoparticles. In 631 all these areas, dynamic test procedures might generate more reliable findings than 632 non-physiological static test conditions. For this reason, the DynaMiTES is an 633 important contribution to pharmaceutical substance testing and may reduce the 634 prevailing risk of late stage drug failures.

635 In addition to the easy adaption of the $\mathrm{HC}$, the concomitant separation of the 636 epithelium and stroma will enable a more precise investigation of substance 637 absorption than previously feasible with the classic HC. By the PC membrane 638 division, it is conceivable that substance accumulation in the two cell compartments 639 can be investigated independently within or after the experiment. In this way, more 640 pharmacokinetic information of the drug absorption can be obtained.

641 Nevertheless, further improvements of the Ocular DynaMiTES will focus on the 642 implementation of online analysis of the acceptor concentration via an inbuilt sensor. 643 Unfortunately, online sensors for this use would require high sensitivity. Thus, 
644 external analysis must be maintained until sufficient technology becomes available.

645 Another technical improvement would be downsizing the compartments to reduce the 646 required substance amount. This is specifically desirable in the preclinical phases 647 when few drug is assessable. However, effects of substance distribution and 648 elimination by tear flow would be more in vivo-like if the volume of the compartments 649 is reduced.

650 Considering the cellular aspects, an investigation of different flow profiles may 651 enhance the comprehension of ocular drug absorption. Furthermore, dynamic 652 cultivation and its effect on the epithelial barrier should be examined. Taken together, 653 these technical and cellular improvements may provide a correlation of the in vitro 654 results with clinical findings. In summary, the present study underlines the particular 655 value of our novel DynaMiTES system and our successful aim to raise the 656 prevalidated $\mathrm{HC}$ to a new sustainable level in in vitro drug absorption testing.

\section{Acknowledgements}

658 The author NB was funded by the Niedersächsisches Ministerium für Wissenschaft 659 und Kultur (MWK) in the joint project SynFoBiA - "Novel synthesis and formulation 660 methods for poorly soluble drugs and sensitive biopharmaceuticals" and the author $661 \mathrm{KM}$ by the Graduate Program $\mu$-Props - "Processing of Poorly Soluble Drugs at 662 Small Scale" within the scope of the Center of Pharmaceutical Engineering (PVZ) of 663 the TU Braunschweig.

664 The authors wish to thank Dr. M. Zorn-Kruppa (Hamburg, Germany) and Dr. K. Araki665 Sasaki (Kagoshima Japan) for their generous gifts of the HCK and HCE-T cell lines. 666 Furthermore, we would like to express our gratitude to Ulrike Kruse for her technical 667 assistance in cell culture and histology. 


\section{Captions}

669 Figure 1: Images of the DynaMiTES; A: The assembled DynaMiTES; B: The three 670 separate levels (from left to right): top level, insert level and bottom level.

671 Figure 2: Depiction of the experimental setup for donor flow or donor dilution 672 experiments; sodium fluorescein donor (yellow) was pumped through the 673 DynaMiTES by applying different flow rates. The permeated amount was measured 674 in the acceptor (light blue). For a clearer overview the incubator is not shown, 675 however, all experiments were performed at $37^{\circ} \mathrm{C}$.

676 Figure 3: Comparison of classic (left; following [20]) and inverted (right) cultivation; 677 light microscopic images show the multilayered epithelium at day 10 of cultivation, 678 which was induced by air-liquid interface (ALI).

679 Figure 4: Cell viability assessed by MTT dye reaction of classic hemicorneas, 680 inverted hemicorneas and stroma equivalents (without HCE-T cells) at day 10 of 681 cultivation; classic hemicorneas were set to $100 \%$ (mean \pm SD.; $n=7-10$; $682 * * p<0.01)$

683 Figure 5: TEER profiles of classic and inverted hemicorneas from day 6 to day 13 of 684 cultivation; air-liquid interface cultivation from day 7 on is marked by a light-blue 685 frame (mean $\left.\pm S D ; n=3-8 ;{ }^{* *} p<0.01\right)$.

686 Figure 6: Comparative permeation study of classic and inverted hemicornea (HC); 687 A: Experimental setup for classic (top) and inverted (bottom) HC with sodium 688 fluorescein donor solution (yellow) and KRB acceptor solution (light blue); yellow 689 arrows mark the direction of sodium fluorescein permeation; B: Resulting apparent 
690

691

692 Figure 7: Cell viability determined by MTT dye reaction after 180 min with and without 693 flow exposition; the negative control (KRB) was kept in a standard well plate and set 694 to $100 \%$. The samples of $0 \mu \mathrm{L} / \mathrm{min}$ were inserted into the DynaMiTES and the 695

710 the lower permeation profiles of $A($ mean $\pm S D ; n=2-6)$.

711 Table 1: Cell morphology of inverted human hemicornea at day 10 of cultivation with

$7121 \mathrm{~h}, 3 \mathrm{~h}$ or $18 \mathrm{~h}$ (overnight) epithelial cell attachment during fabrication; "hair-like" 
713 structures in the epithelium resulted from the known but unavoidable interaction of 714 the PC membrane and resin material.

715 Table 2: Images of light microscopy (left) and scanning electron microscopy (right) of 716 inverted hemicorneas after 180 min with and without flow; the negative control was 717 kept in a standard well plate $(0 \mu \mathrm{L} / \mathrm{min}$ well plate). The samples of $0 \mu \mathrm{L} / \mathrm{min}$ were 718 inserted into the DynaMiTES and the system was filled only once $(0 \mu \mathrm{L} / \mathrm{min}$ 719 DynaMiTES). For the positive control, inverted HCs were scratched with a glass 720 pipette. The injured area with the visible PC membrane is marked in red.

\section{$721 \quad 13$ References}

722 [1] American Academy of Ophthalmology, Fundamentals and Principles of 723 Ophthalmology: Part 3: Biochemistry and Metabolism, American Academy of 724 Ophthalmology, 1989.

725 [2] K. Araki-Sasaki, Y. Ohashi, T. Sasabe, K. Hayashi, H. Watanabe, Y. Tano, H. 726 Handa, An SV40-immortalized human corneal epithelial cell line and its characterization, Investigative Ophthalmology and Visual Science 36 (1995) 614621.

729 [3] B. Ataç, I. Wagner, R. Horland, R. Lauster, U. Marx, A.G. Tonevitsky, R.P. Azar, 730 G. Lindner, Skin and hair on-a-chip: In vitro skin models versus ex vivo tissue 731 maintenance with dynamic perfusion, Lab on a Chip - Miniaturisation for Chemistry and Biology 13 (2013) 3555-3561.

733 [4] J. Barar, A.R. Javadzadeh, Y. Omidi, Ocular novel drug delivery: Impacts of membranes and barriers, Expert Opinion on Drug Delivery 5 (2008) 567-581. 
735 [5] N. Beißner, T. Lorenz, S. Reichl, Organ on Chip, in: A. Dietzel (Ed.), 736 Microsystems for Pharmatechnology: Manipulation of Fluids, Particles, Droplets, 737 and Cells, 1st ed., Springer, 2016, pp. 299-339.

738 [6] R. Booth, H. Kim, Characterization of a microfluidic in vitro model of the blood739 740

[7] M. Bur, B. Rothen-Rutishauser, H. Huwer, C.-M. Lehr, A novel cell compatible 742 impingement system to study in vitro drug absorption from dry powder aerosol formulations, European Journal of Pharmaceutics and Biopharmaceutics 72 (2009) 350-357.

[8] N.L. Burstein, Preservative alteration of corneal permeability in humans and rabbits, Investigative ophthalmology \& visual science 25 (1984) 1453.

[9] O. Camber, P. Edman, Influence of some preservatives on the corneal permeability of pilocarpine and dexamethasone, in vitro, International Journal of Pharmaceutics 39 (1987) 229-234.

[10] A.K. Capulli, K. Tian, N. Mehandru, A. Bukhta, S.F. Choudhury, M. Suchyta, K.K. Parker, Approaching the in vitro clinical trial: Engineering organs on chips, Lab on a Chip - Miniaturisation for Chemistry and Biology 14 (2014) 3181-3186.

[11] W. Chen, Z. Li, J. Hu, Z. Zhang, L. Chen, Y. Chen, Z. Liu, Corneal alternations induced by topical application of benzalkonium chloride in rabbit, PLoS ONE 6 (2011).

[12] C. Chow, J.P. Gilbard, Tear Film, in: J.H. Krachmer, M.J. Mannis, E.J. Holland (Eds.), Cornea: Fundamentals of Cornea and External Disease, 1st ed., Mosby, St. Louis, Mo., 1997, pp. 49-60. 
759 [13] S.S. Chrai, T.F. Patton, A. Mehta, J.R. Robinson, Lacrimal and instilled fluid 760 dynamics in rabbit eyes, Journal of Pharmaceutical Sciences 62 (1973) 1112$761 \quad 1121$.

762 [14] J.P. Craig, A. Tomlison, L. McCann, Tear Film, in: D.A. Dartt (Ed.), 763 Encyclopedia of the eye, Elsevier Acad. Press, Amsterdam, 2010, pp. 254-262.

764 [15] P.N. Dilly, Structure and function of the tear film, in: D.A. Sullivan (Ed.), 765 Lacrimal Gland, Tear Film and Dry Eye Syndroms: Basic Science and Clinical 766 Relevance, Plenum Press, New York and London, 1994, pp. 239-247.

767 [16] M.H. Friedlaender, D. Breshears, H. Sheardown, B. Amoozgar, M. Senchyna, 768 The dilution of benzalkonium chloride (BAK) in the tear film, Advances in Therapy $76923(2006) 835-841$.

770 [17] K. Green, The effects of preservatives on corneal permeability of drugs, in: P. 771 Edman (Ed.), Biopharmaceutics of ocular drug delivery, CRC Press, Boca Raton, $772 \quad$ Fla, 1993, pp. 43-59.

773 [18] K. Green, A.M. Tonjum, The effect of benzalkonium chloride on the 774 electropotential of the rabbit cornea, Acta Ophthalmologica 53 (1975) 348-357.

775 [19] M. Hahne, S. Reichl, Development of a serum-free human cornea construct 776 for in vitro drug absorption studies: The influence of varying cultivation parameters 777 on barrier characteristics, International Journal of Pharmaceutics 416 (2011) 2682778.

779 [20] M. Hahne, M. Zorn-Kruppa, G. Guzman, J.M. Brandner, E. Haltner-Ukomado, 780 H. Wätzig, S. Reichl, Prevalidation of a human cornea construct as an alternative 781 to animal corneas for in vitro drug absorption studies, Journal of Pharmaceutical $782 \quad$ Sciences $101(2012)$ 2976-2988.

783 [21] M. Hornof, E. Toropainen, A. Urtti, Cell culture models of the ocular barriers, $784 \quad$ Eur. J. Pharm. Biopharm. 60 (2005) 207-225. 
785

786

787

788

[22] D. Huh, B.D. Matthews, A. Mammoto, M. Montoya-Zavala, H. Yuan Hsin, D.E. Ingber, Reconstituting organ-level lung functions on a chip, Science 328 (2010) $1662-1668$.

[23] K. Järvinen, T. Järvinen, A. Urtti, Ocular absorption following topical delivery, Advanced Drug Delivery Reviews 16 (1995) 3-19.

[24] I.P. Kaur, R. Smitha, Penetration Enhancers and Ocular Bioadhesives: Two New Avenues for Ophthalmic Drug Delivery, Drug Development and Industrial Pharmacy 28 (2002) 353-369.

[25] H.J. Kim, D. Huh, G. Hamilton, D.E. Ingber, Human gut-on-a-chip inhabited by microbial flora that experiences intestinal peristalsis-like motions and flow, Lab on a Chip - Miniaturisation for Chemistry and Biology 12 (2012) 2165-2174.

[26] Kirkstall Ltd., homepage, http://kirkstall.org/index.php/quasi-vivo-products/, accessed 14 December 2016.

[27] C. Kölln, S. Reichl, Cytochrome P450 Activity in Ex Vivo Cornea Models and a Human Cornea Construct, Journal of Pharmaceutical Sciences 105 (2016) 22042212.

[28] C. Kölln, S. Reichl, Expression of glutathione transferases in corneal cell lines, corneal tissues and a human cornea construct, International Journal of Pharmaceutics 506 (2016) 371-381.

[29] M. Kusano, M. Uematsu, T. Kumagami, H. Sasaki, T. Kitaoka, Evaluation of acute corneal barrier change induced by topically applied preservatives using corneal transepithelial electric resistance in vivo, Cornea 29 (2010) 80-85.

[30] L. Maciuleviciute, C. Hornberg, N.H. Seemayer, Rodent tracheal epithelial cells in vitro: A comparative study of normal cells, enhanced growth variants, SV40 transformed cells and their interactions, Toxicology Letters 88 (1996) 55-64. 
810 [31] S. Maher, L. Feighery, D.J. Brayden, S. McClean, Melittin as a permeability 811 enhancer II: in vitro investigations in human mucus secreting intestinal 812 monolayers and rat colonic mucosae, Pharmaceutical Research 24 (2007) 1346$813 \quad 1356$.

814 [32] U. Marx, H. Walles, S. Hoffmann, G. Lindner, R. Horland, F. Sonntag, U. 815 Klotzbach, D. Sakharov, A. Tonevitsky, R. Lauster, 'Human-on-a-chip' 816 developments: A translational cuttingedge alternative to systemic safety 817 assessment and efficiency evaluation of substances in laboratory animals and 818 man?, ATLA Alternatives to Laboratory Animals 40 (2012) 235-257.

819 [33] S. Mishima, A. Gasset, S.D. Klyce, Baum, J. L. [JR.], Determination of tear $820 \quad$ volume and tear flow, Investigative ophthalmology 5 (1966) 264-276.

821 [34] S. Nakagawa, T. Usui, S. Yokoo, S. Omichi, M. Kimakura, Y. Mori, K. Miyata, 822 M. Aihara, S. Amano, M. Araie, Toxicity evaluation of antiglaucoma drugs using 823 stratified human cultivated corneal epithelial sheets, Investigative Ophthalmology $824 \quad$ and Visual Science 53 (2012) 5154-5160.

825 [35] T. Nakamura, M. Teshima, T. Kitahara, H. Sasaki, M. Uematsu, T. Kitaoka, M. 826 Nakashima, K. Nishida, J. Nakamura, S. Higuchi, Sensitive and real-time method 827 for evaluating corneal barrier considering tear flow, Biological and Pharmaceutical $828 \quad$ Bulletin $33(2010)$ 107-110.

829 [36] T. Nakamura, M. Yamada, M. Teshima, M. Nakashima, H. To, N. Ichikawa, H. 830 Sasaki, Electrophysiological characterization of tight junctional pathway of rabbit 831 cornea treated with ophthalmic ingredients, Biological and Pharmaceutical Bulletin 832 30 (2007) 2360-2364.

833 [37] I. Pepić, J. Lovrić, B. Cetina-Čižmek, S. Reichl, J. Filipović-Grčić, Toward the 834 practical implementation of eye-related bioavailability prediction models, Drug 835 Discovery Today 19 (2014) 31-44. 
836 [38] S. Pretor, J. Bartels, T. Lorenz, K. Dahl, J.H. Finke, G. Peterat, R. Krull, A.T. 837 Al-Halhouli, A. Dietzel, S. Büttgenbach, S. Reichl, C.C. Müller-Goymann, Cellular 838 uptake of coumarin-6 under microfluidic conditions into HCE-T cells from 839 nanoscale formulations, Molecular Pharmaceutics 12 (2015) 34-45.

840 [39] K.J. Regehr, M. Domenech, J.T. Koepsel, K.C. Carver, S.J. Ellison-Zelski, 841 W.L. Murphy, L.A. Schuler, E.T. Alarid, D.J. Beebe, Biological implications of 842 polydimethylsiloxane-based microfluidic cell culture, Lab on a Chip Miniaturisation for Chemistry and Biology 9 (2009) 2132-2139.

[40] S. Reichl, Cell culture models of the human cornea - A comparative evaluation of their usefulness to determine ocular drug absorption in-vitro, Journal of Pharmacy and Pharmacology 60 (2008) 299-307.

847 [41] H. Sasaki, K. Yamamura, C. Tei, K. Nishida, J. Nakamura, Ocular permeability of FITC-dextran with absorption promoter for ocular delivery of peptide drug, Journal of Drug Targeting 3 (1995) 129-135.

[42] J.W. Shell, Pharmacokinetics of topically applied ophthalmic drugs, Survey of $851 \quad$ Ophthalmology 26 (1982) 207-218.

852 [43] T. Sörensen, F. Taagehöj Jensen, Tear flow in normal human eyes. 853 determination by means of radioisotope and gamma camera, Acta Ophthalmologica 57 (1979).

[44] G.I. Tennekoon, J. Yoshino, K. Peden, J. Bigbee, J.L. Rutkowski, Y. Kishimoto, G.H. DeVries, G.M. McKhann, Transfection of neonatal rat Schwann cells with SV-40 large $\mathrm{T}$ antigen gene under control of the metallothionein promoter, Journal of Cell Biology 105 (1987) 2315-2325.

[45] M.W. Toepke, D.J. Beebe, PDMS absorption of small molecules and consequences in microfluidic applications, Lab on a Chip - Miniaturisation for Chemistry and Biology 6 (2006) 1484-1486. 
[46] A.M. Tonjum, Permeability of rabbit corneal epithelium to horseradish peroxidase after the influence of benzalkonium chloride, Acta Ophthalmologica 53 (1975) 335-347.

[47] M. Uematsu, Y.H. Mohamed, N. Onizuka, R. Ueki, D. Inoue, A. Fujikawa, H. Sasaki, T. Kitaoka, Less Invasive Corneal Transepithelial Electrical Resistance Measurement Method, Ocular Surface 14 (2016) 37-42.

[48] T. Utsunomiya, A. Ishibazawa, T. Nagaoka, K. Hanada, H. Yokota, N. Ishii, A. Yoshida, Transforming growth factor-B signaling cascade induced by mechanical stimulation of fluid shear stress in cultured corneal epithelial cells, Investigative Ophthalmology and Visual Science 57 (2016) 6382-6388.

[49] M.W. Van Der Helm, A.D. Van Der Meer, Eijkel, J. C. T., A. Van Den Berg, L.I.

[50] P.M. Van Midwoud, G. Groothuis, M.T. Merema, E. Verpoorte, Microfluidic biochip for the perifusion of precision-cut rat liver slices for metabolism and toxicology studies, Biotechnology and Bioengineering 105 (2010) 184-194.

[51] P.M. Van Midwoud, A. Janse, M.T. Merema, G. Groothuis, E. Verpoorte, Segerink, Microfluidic organ-on-chip technology for blood-brain barrier research, Tissue barriers 4 (2016) e1142493.

[52] J. Verstraelen, S. Reichl, Expression analysis of MDR1, BCRP and MRP3 transporter proteins in different in vitro and ex vivo cornea models for drug absorption studies, International Journal of Pharmaceutics 441 (2013) 765-775.

[53] J. Verstraelen, S. Reichl, Multidrug resistance-associated protein (MRP1, 2, 4 and 5) expression in human corneal cell culture models and animal corneal tissue, Molecular Pharmaceutics 11 (2014) 2160-2171. 
888 [54] J. Verstraelen, S. Reichl, Upregulation of P-glycoprotein expression by 889 ophthalmic drugs in different corneal in-vitro models, The Journal of pharmacy $890 \quad$ and pharmacology 67 (2015) 605-615.

891 [55] I. Wagner, E.-M. Materne, S. Brincker, U. Süßbier, C. Frädrich, M. Busek, F. 892 Sonntag, D.A. Sakharov, E.V. Trushkin, A.G. Tonevitsky, R. Lauster, U. Marx, A 893 dynamic multi-organ-chip for long-term cultivation and substance testing proven 894 by $3 \mathrm{D}$ human liver and skin tissue co-culture, Lab on a Chip - Miniaturisation for $895 \quad$ Chemistry and Biology 13 (2013) 3538-3547.

896 [56] Y. Wakabayashi, J. Chua, J.M. Larkin, J. Lippincott-Schwartz, I.M. Arias, Four897 dimensional imaging of filter-grown polarized epithelial cells, Histochemistry and $898 \quad$ Cell Biology 127 (2007) 463-472.

899 [57] M. Zorn-Kruppa, S. Tykhonova, G. Beige, J. Bednarz, H.A. Diehl, M. Engelke, 900 A human corneal equivalent constructed from SV40-immortalised corneal cell $901 \quad$ lines, Alternatives to Laboratory Animals 33 (2005) 37-45. 


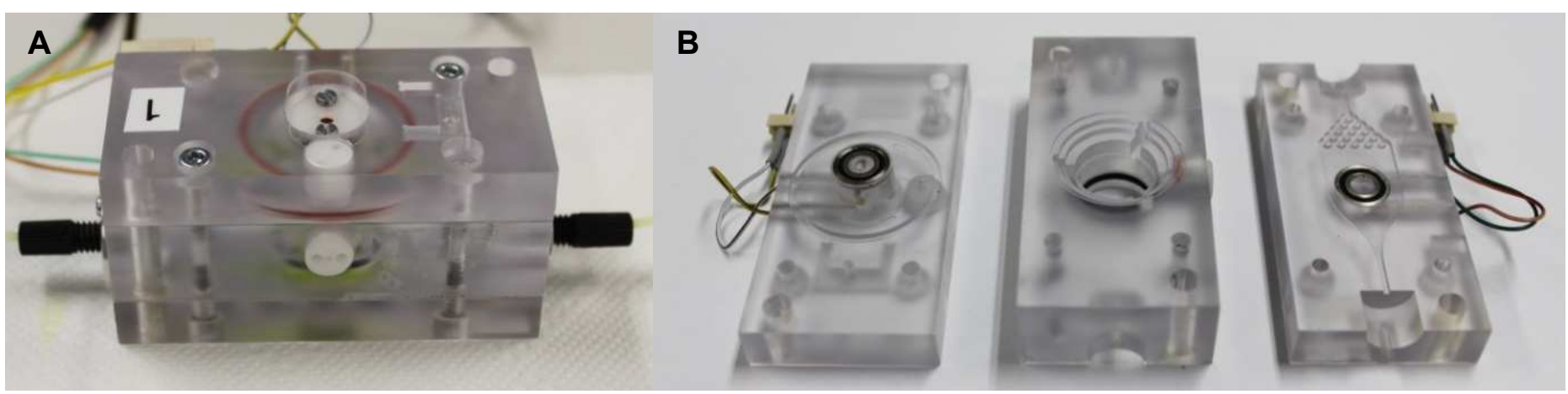




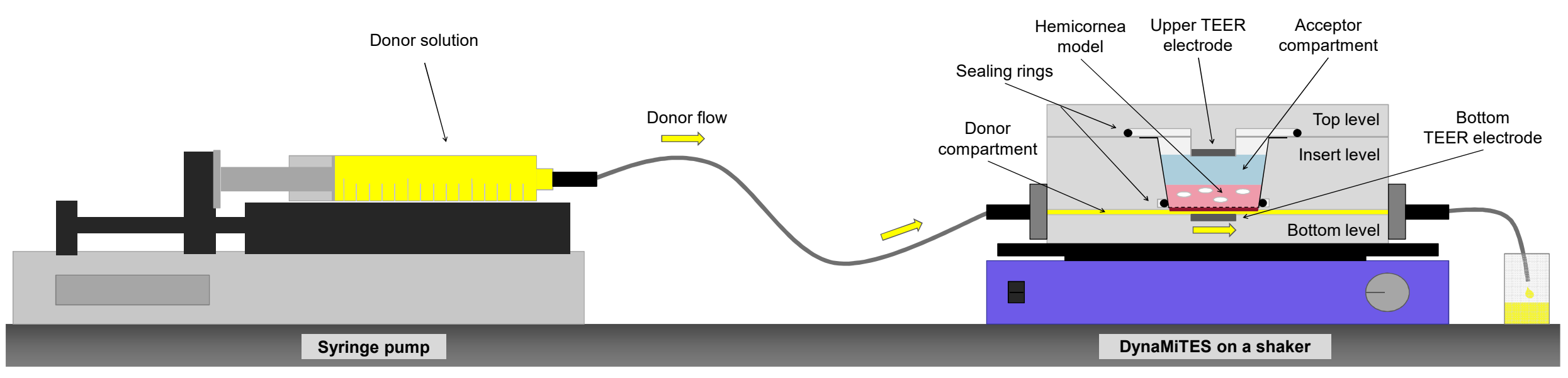




\section{$\underline{\text { Classic Cultivation Inverted Cultivation }}$}

Casting of collagen gel with keratocytes

$(\mathrm{HCK}-)$
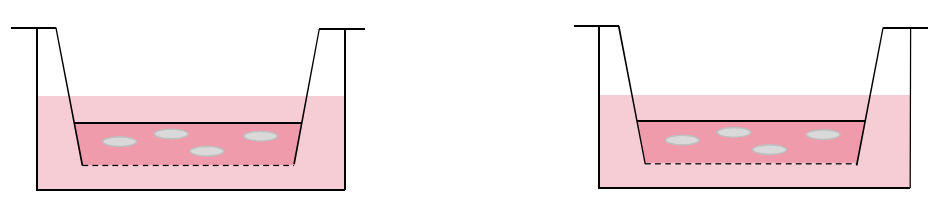

Day 1

Seeding of epithelial cells (HCE-T • )
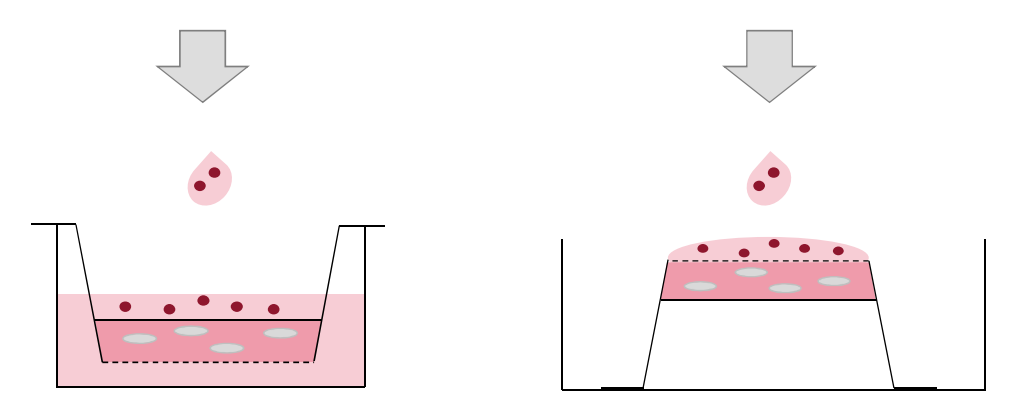

Day 1

Submersed cultivation
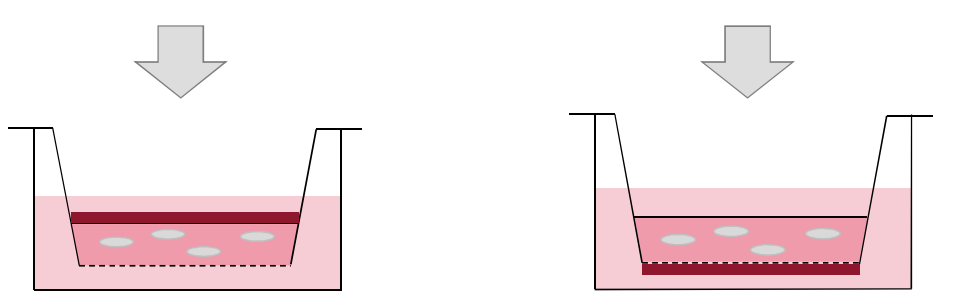

Day 1 to Day 7

Cultivation at air-liquid interface

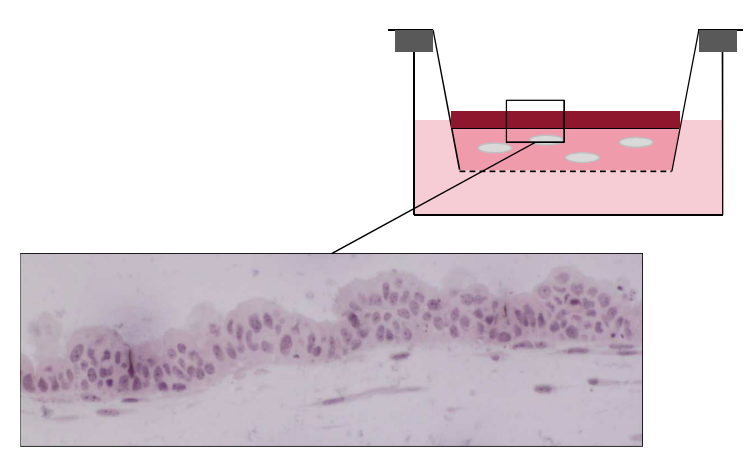

Day 7 to Day 10 


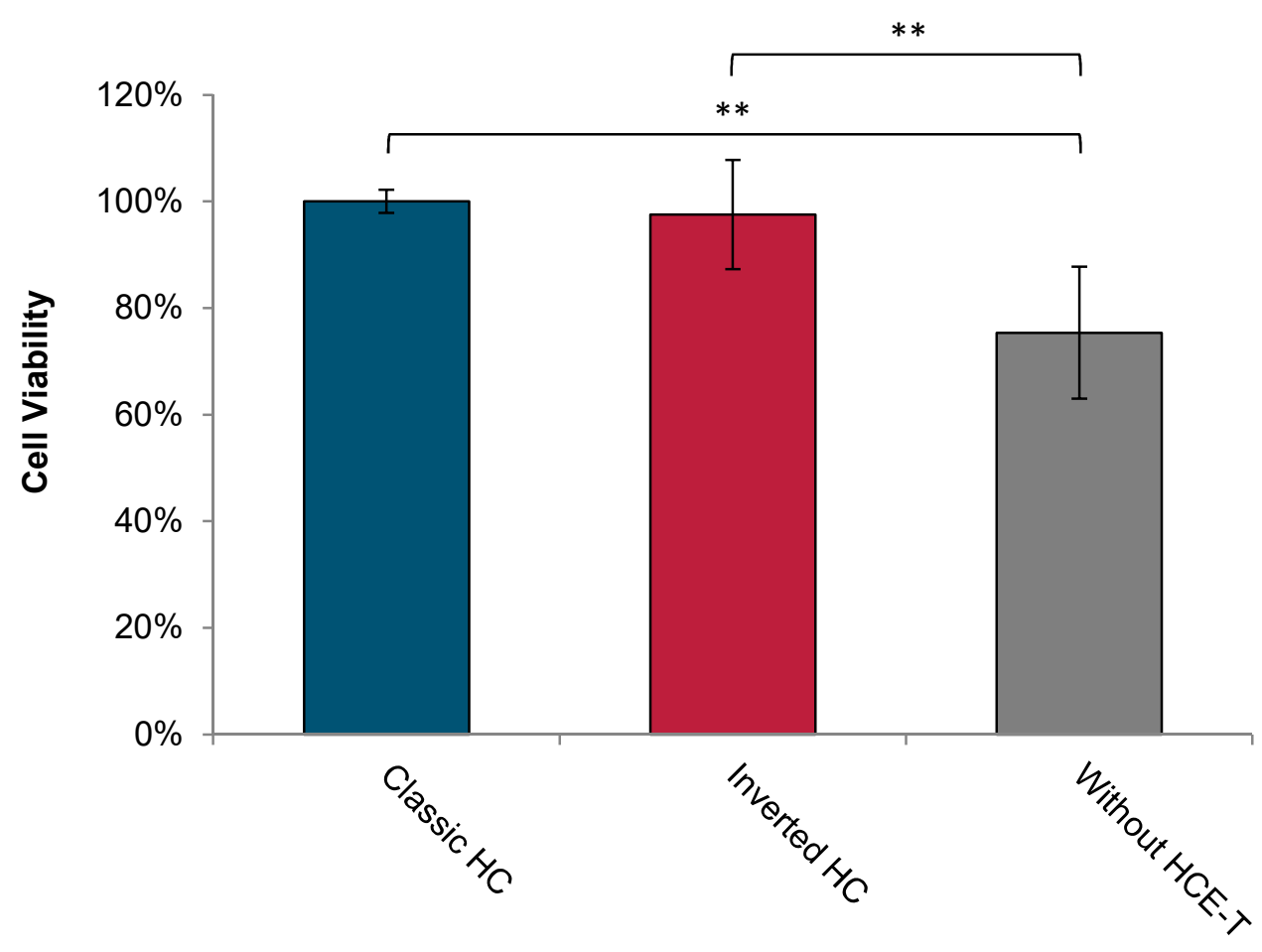




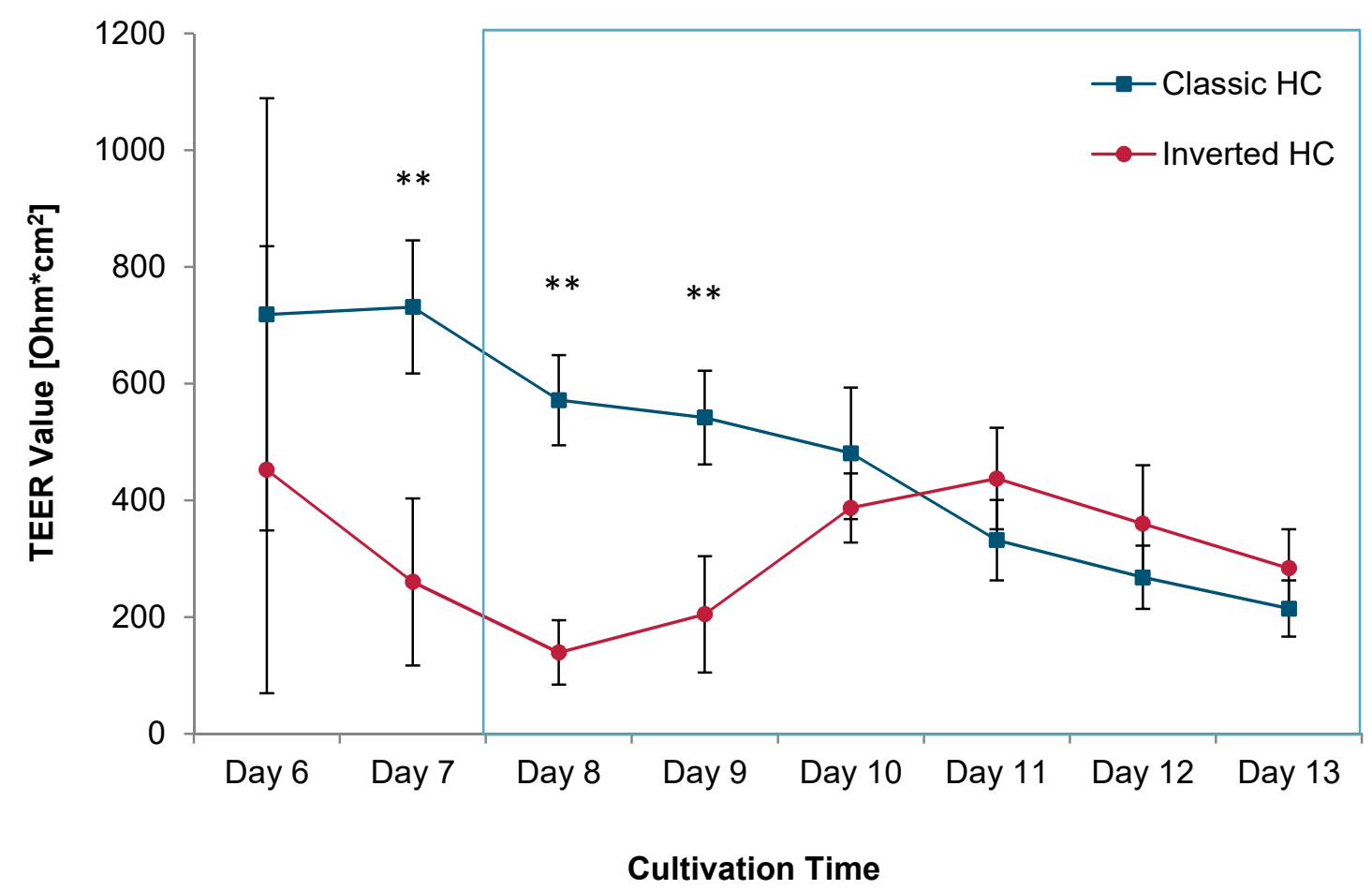


A

\section{Classic HC}

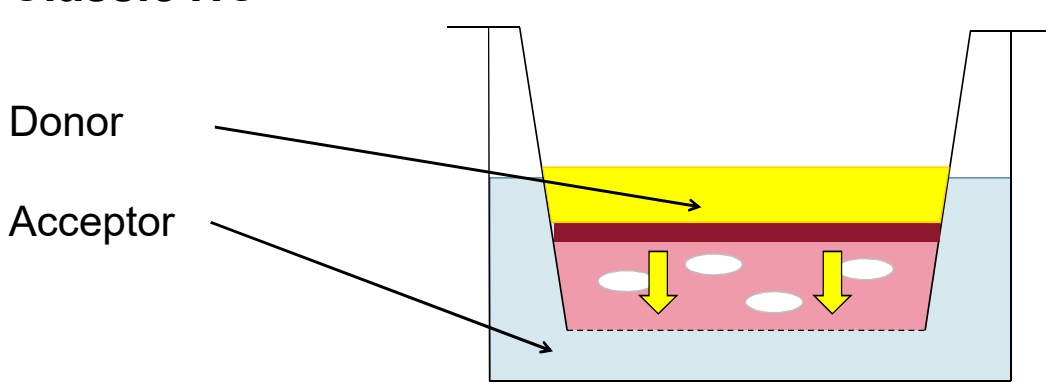

\section{Inverted HC}

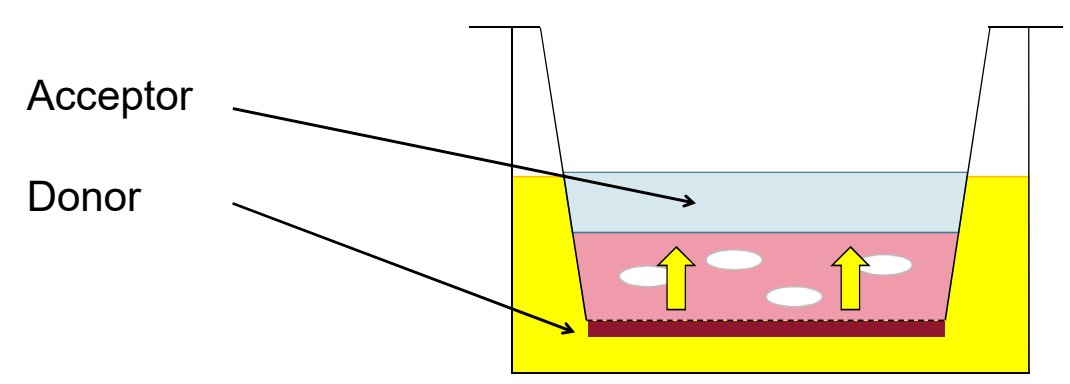

B

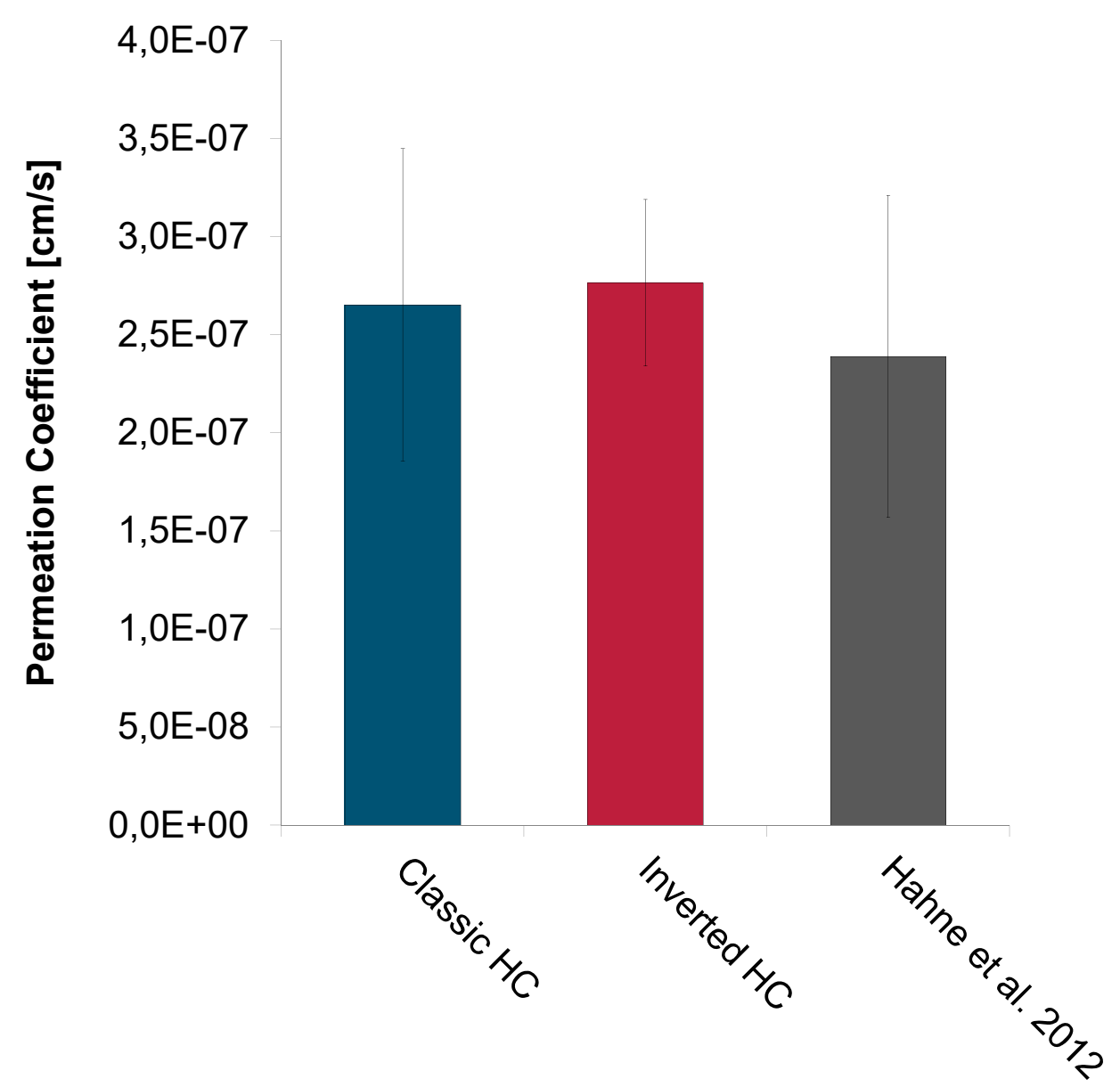




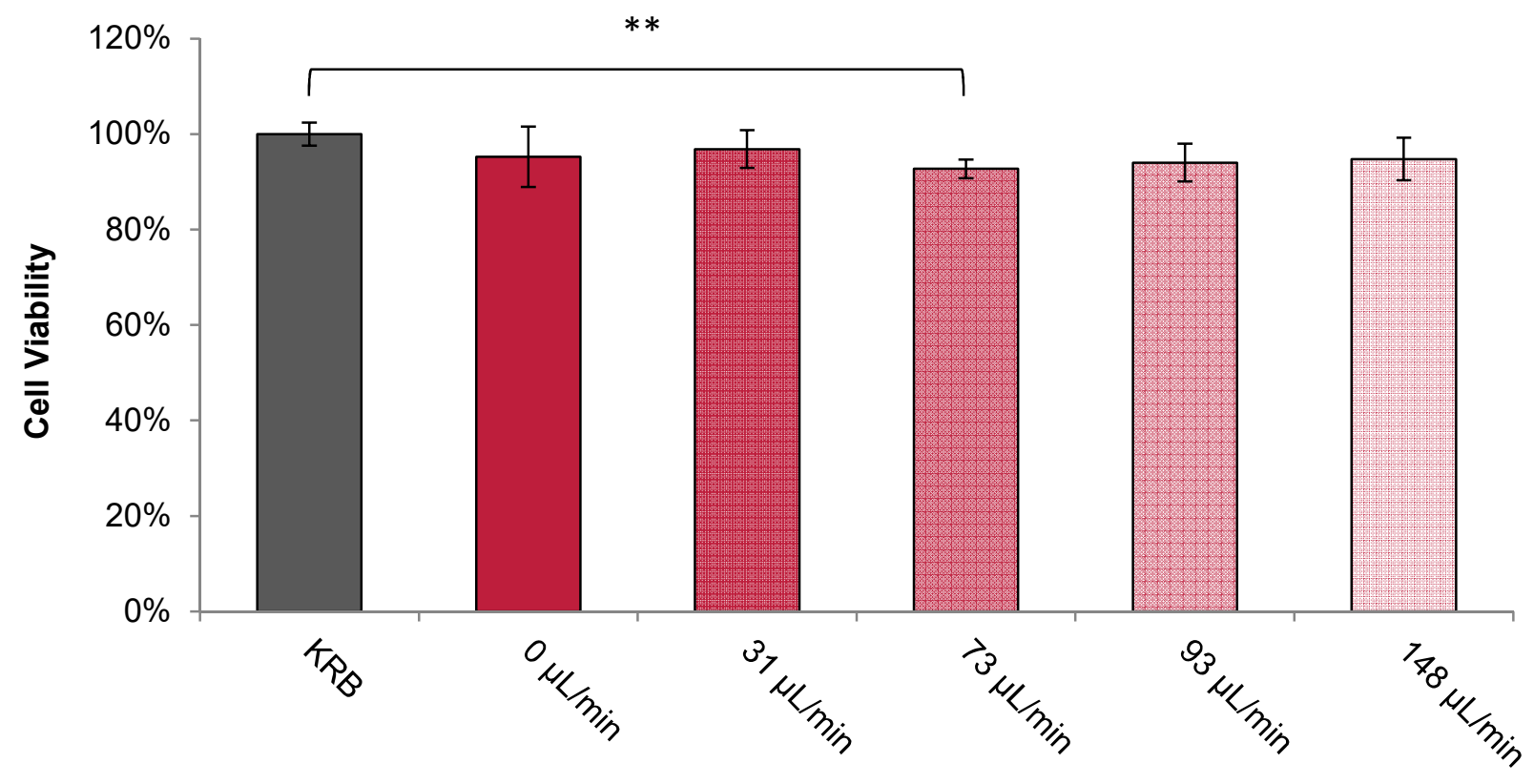




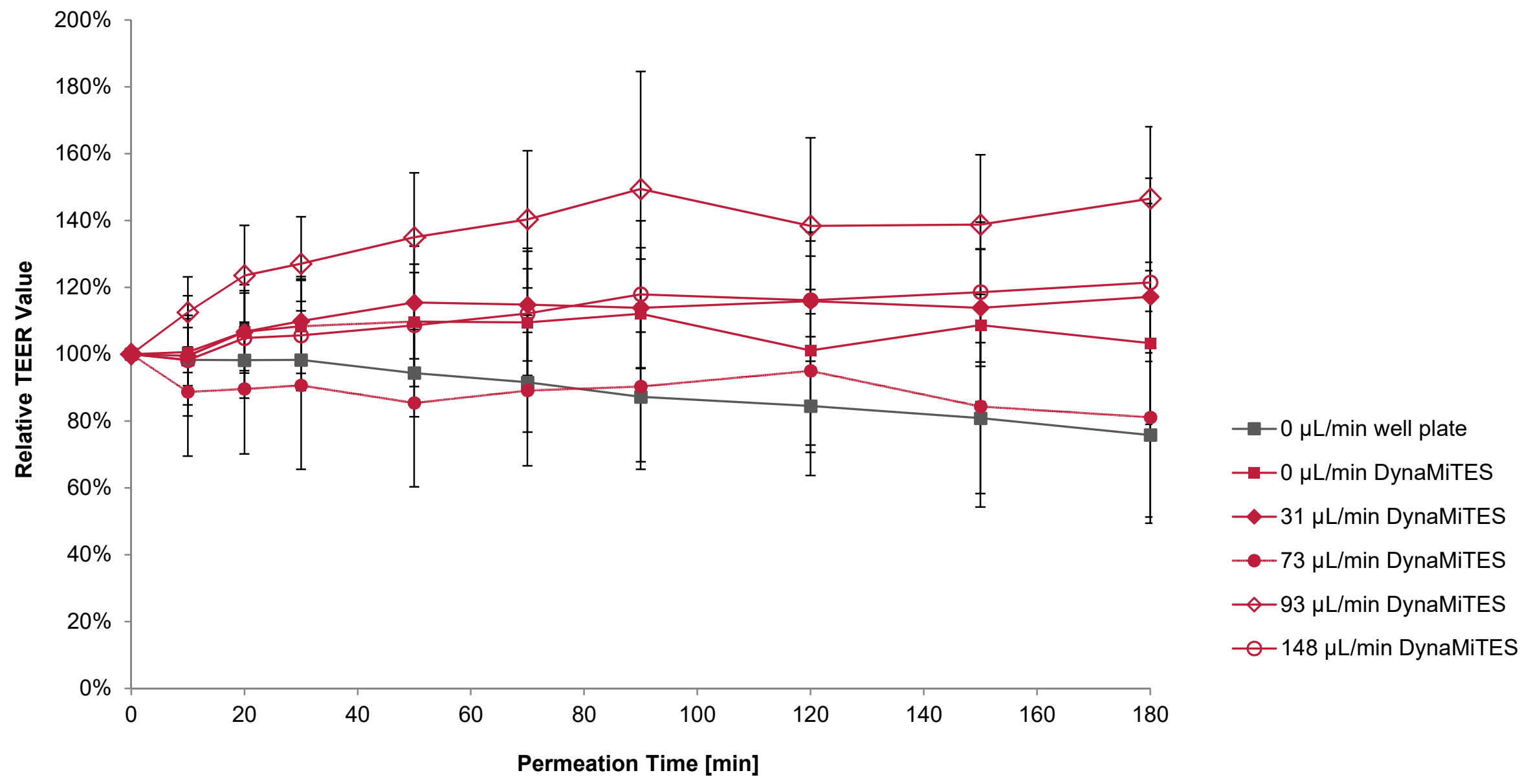




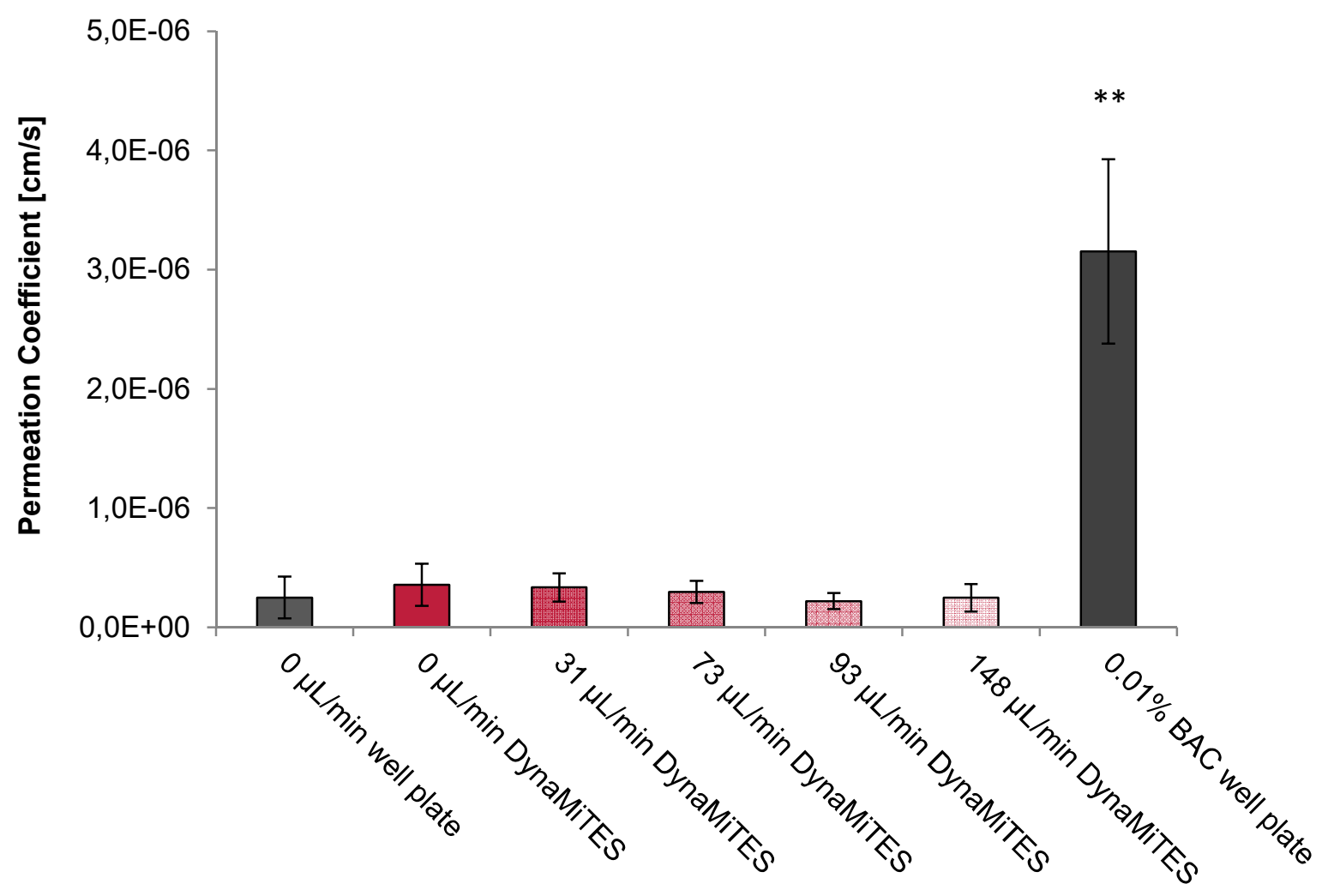



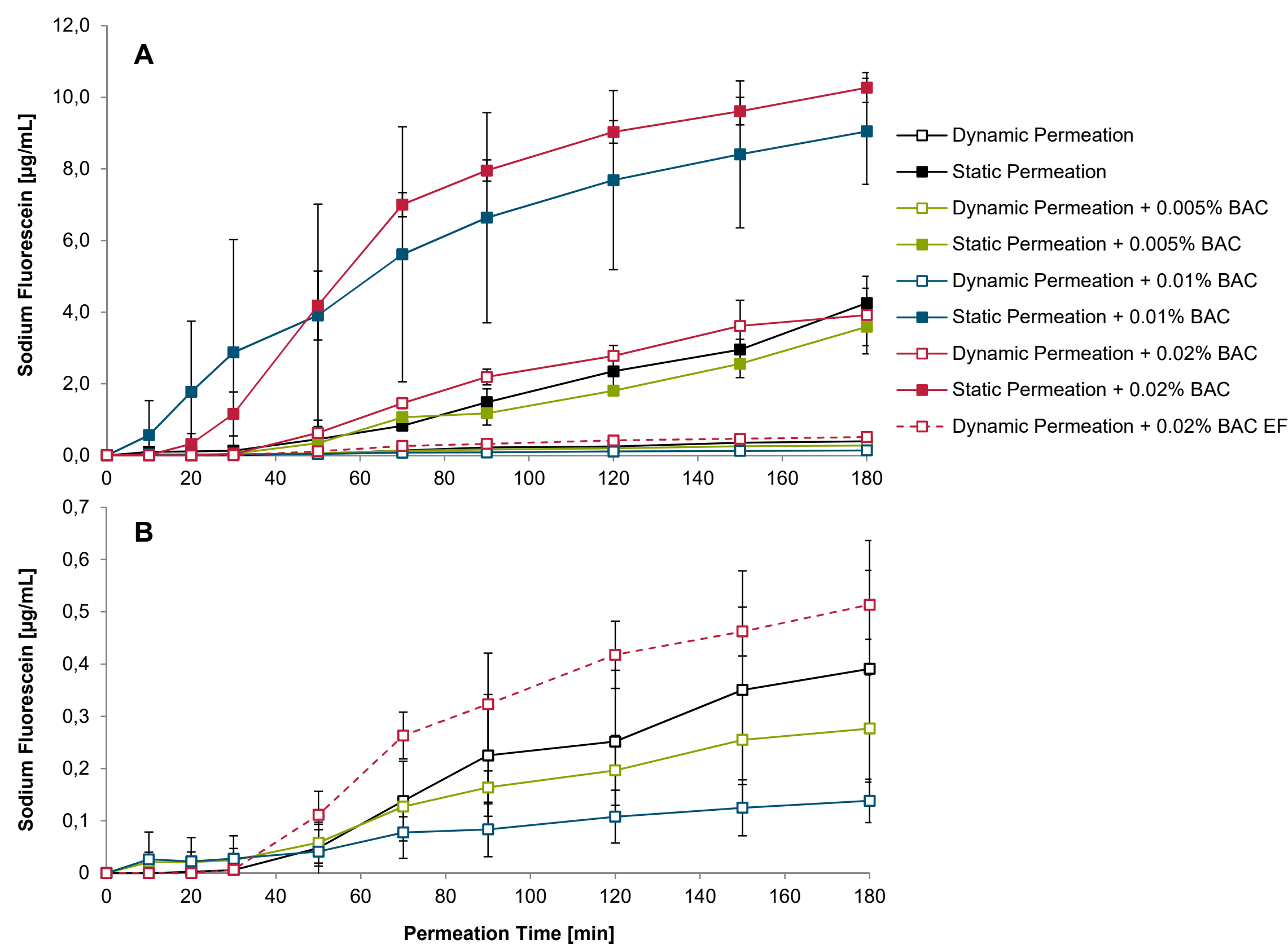


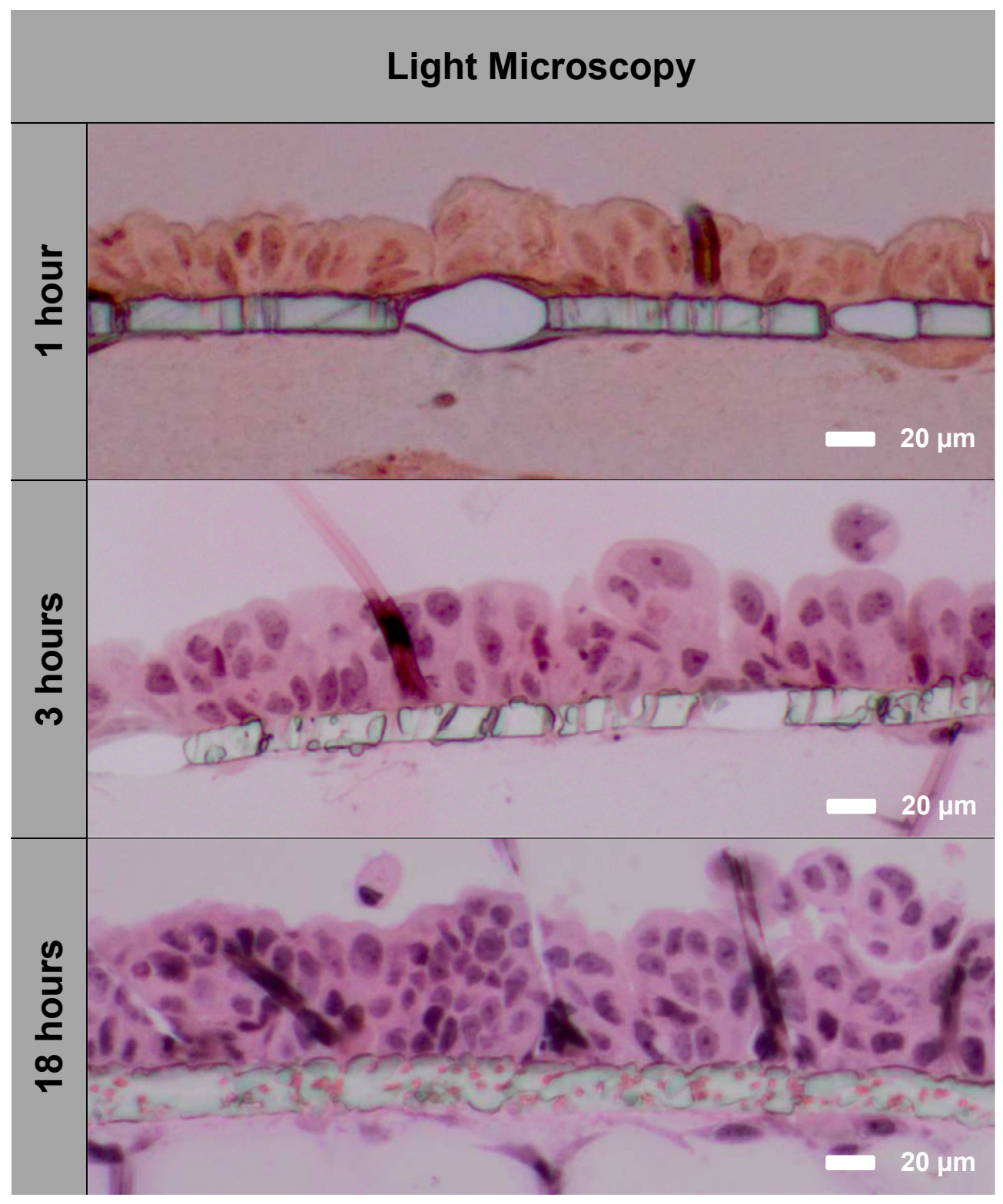




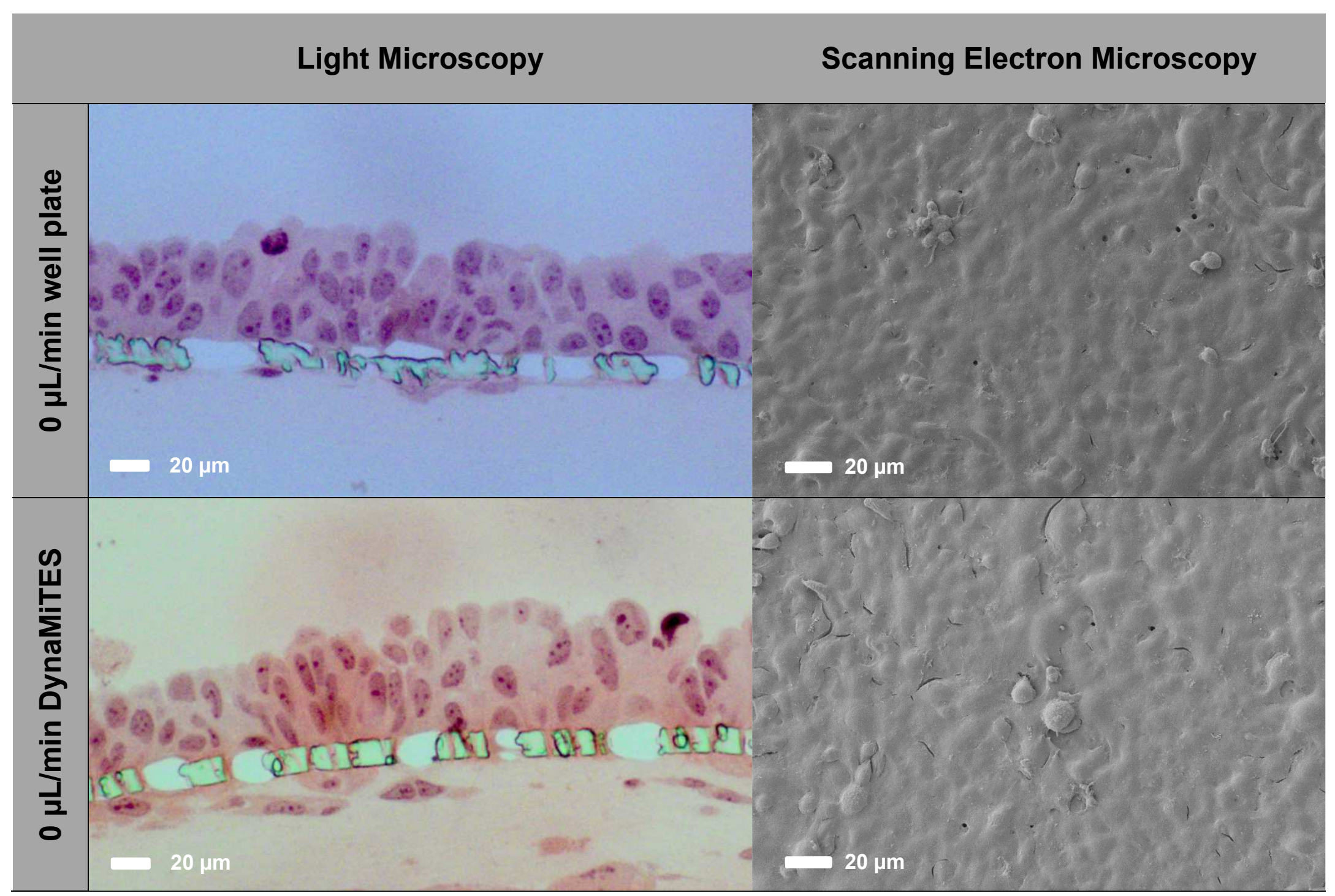




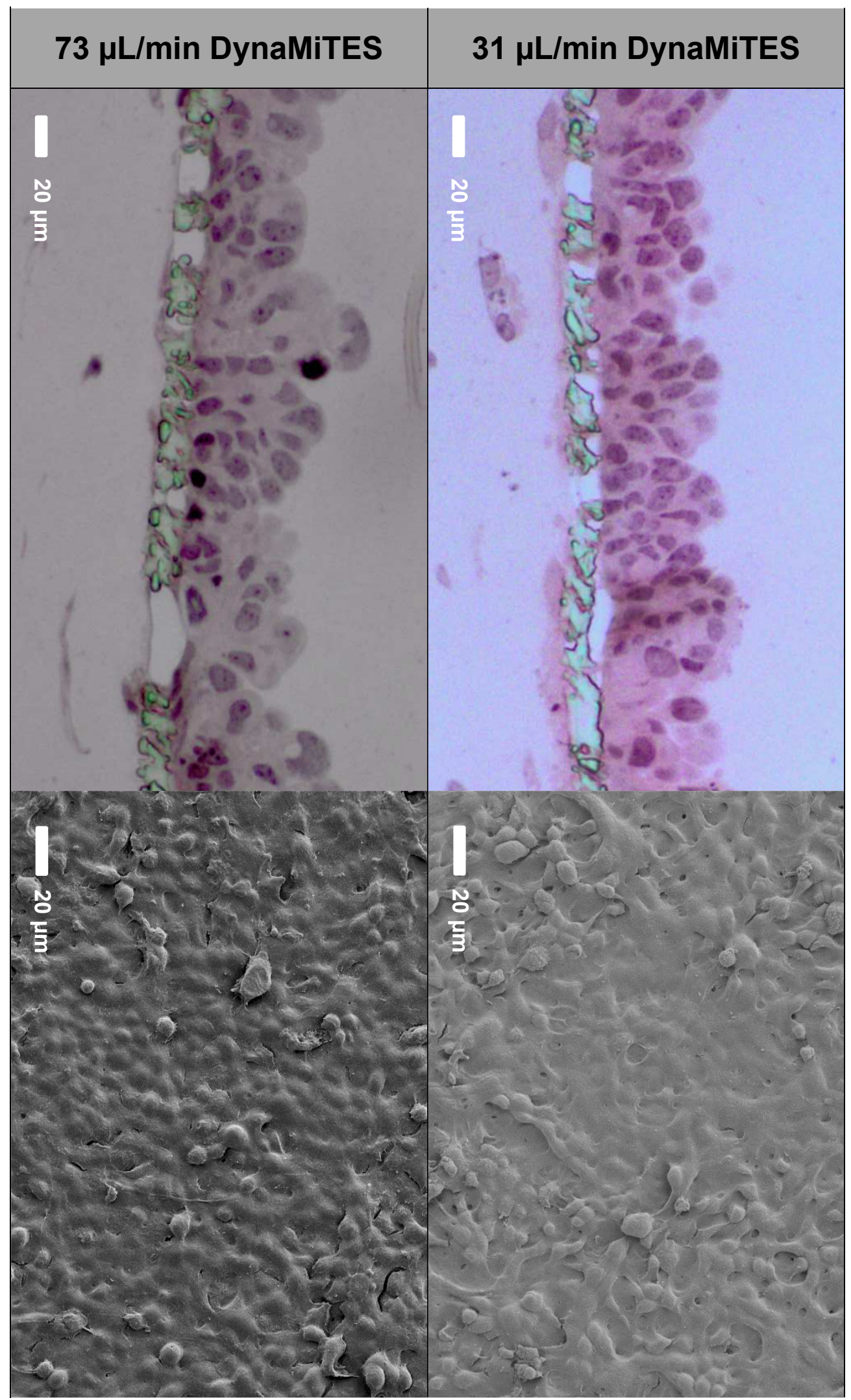




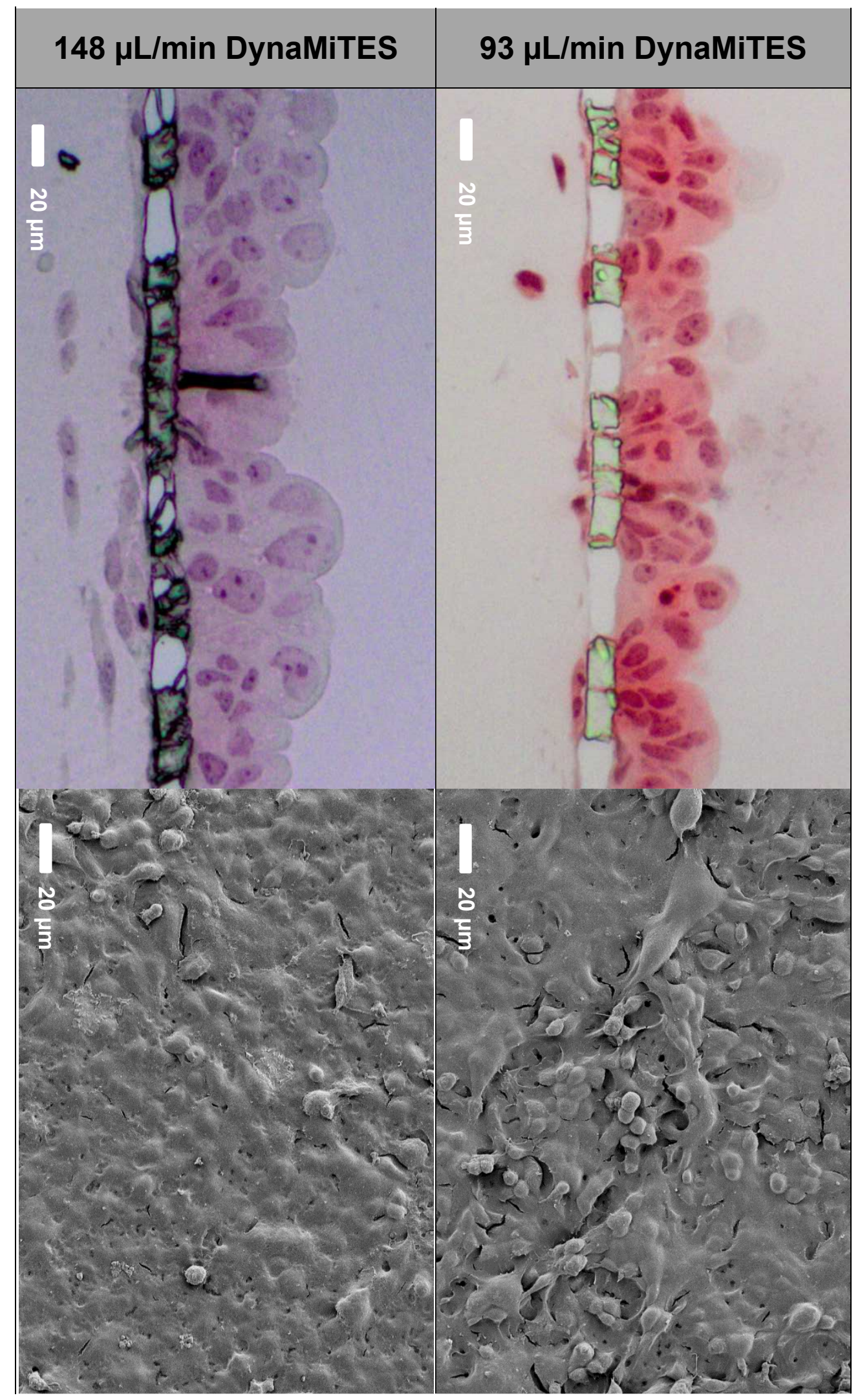




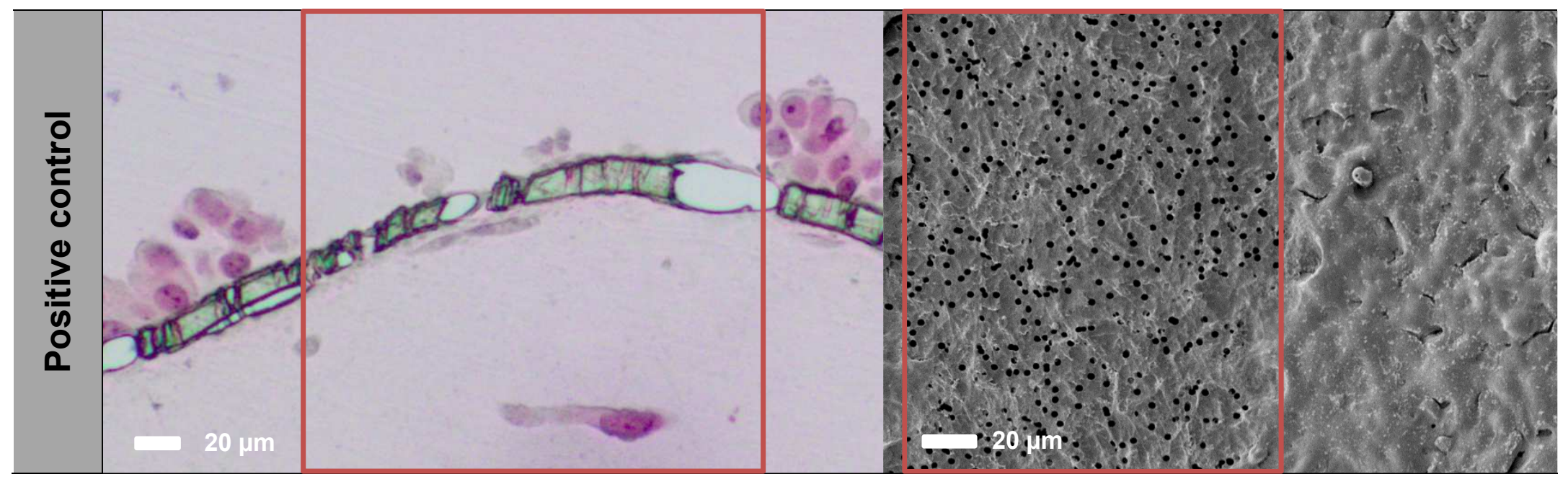

